October 12,2006

\title{
AN X-RAY AND NEAR-INFRARED STUDY OF YOUNG STARS IN THE CARINA NEBULA
}

\author{
Kaushar Sanchawala, Wen-Ping Chen, Hsu-Tai Lee \\ National Central University, Taiwan \\ You-Hua Chu \\ University of Illinois at Urbana Champaign, USA \\ Yasushi Nakajima, Motohide Tamura \\ National Astronomical Observatory of Japan, Japan \\ and \\ Daisuke Baba, Shuji Sato \\ Department of Astrophysics, Nagoya University, Japan
}

\begin{abstract}
We present a multiwavelength study of the central region of the Carina Nebula, including Trumpler 16 and part of Trumpler 14. Our analysis of the Chandra $X$-ray Observatory archival data led to the identification of 454 X-ray sources. These sources were then cross-identified with optical photometric and spectroscopic information available from the literature, and with newly obtained nearinfrared $\left(J H K_{s}\right)$ imaging observations. A total of 38 known OB stars are found to be X-ray emitters. All the $\mathrm{O}$ stars and early-B stars follow the nominal relation between the X-ray and bolometric luminosities, $L_{\mathrm{X}} \sim 10^{-7} L_{\text {bol }}$. A few mid- to late-type B stars are found to be associated with X-ray emission, likely attributable to T Tauri companions. We discovered $16 \mathrm{OB}$ star candidates that suffer a large extinction in the optical wavebands. Some 300 sources have the Xray and infrared characteristics of late-type pre-main sequence stars. Our sample represents the most comprehensive census of the young stellar population in the Carina Nebula so far and should be useful for the study of the star-formation history of this massive starburst region. We also report the finding of a compact
\end{abstract}


This is an unedited preprint of an article accepted for publication in The Astrophysical Journal. The final published article may differ from this preprint. Copyright 2006 by The American Astronomical Society. Please cite as 'ApJ preprint doi:10.1086/'510184".

$\left(2^{\prime} \times 4^{\prime}\right)$ group of 10 relatively bright X-ray sources, all of which are detected in the near-infrared wavelengths and are highly reddened. The group is spatially coincident with the dark ' $V$ ' shaped dust lane bisecting the Carina Nebula, and may be part of an embedded association. The distribution of the young stellar groups surrounding the H II region associated with Trumpler 16 is consistent with the collect-and-collapse scenario of triggered star formation.

Subject headings: ISM: individual (Carina Nebula) — open clusters and associations: individual (Trumpler 16) — stars: early-type — dust, extinction X-rays: stars

\section{INTRODUCTION}

Massive stars have a profound influence on neighboring molecular clouds. The powerful stellar radiation and winds from even a single massive star may sweep away nearby clouds and prevent subsequent star formation. The massive star on the other hand may prompt the collapse of a nearby molecular cloud which otherwise may not contract spontaneously. The Carina Nebula, also known as NGC 3372, is a remarkable star-forming region, where the most massive stars known in the Milky-Way Galaxy co-exist. The Nebula, which occupies approximately a 4 square degree area of the sky, contains at least a dozen known star clusters (Feinstein 1995). The clusters for which photometric and spectroscopic data are available include: Bochum (Bo) 10 and 11, Trumpler (Tr) 14, 15 and 16, Collinder (Cr) 228, NGC 3293 and NGC 3324. Among these, Tr 14 and Tr 16, in the central region of the Nebula, are the youngest and the most populous star clusters. The reported distance modulus for Tr 16 ranges from 11.8 (Levato et al. 1981) to 12.55 (Massey \& Johnson 1993, MJ93 hereafter) and for $\operatorname{Tr} 14$, from 12.20 (Feinstein 1983) to 12.99 (Morrell et al. 1988). Walborn (1995) derived a distance of $2.5 \mathrm{kpc}$ for $\operatorname{Tr} 16$ using $R=3.5$, where $R=A_{V} / E(B-V)$ is the ratio of total to selective extinction. Crowther et al. (1995) derived a distance of $2.6 \mathrm{kpc}$ for $\operatorname{Tr} 14$. Walborn (1973) and Morrell et al. (1988) found the two clusters to be at slightly different distances, whereas Turner \& Moffat (1980) and MJ93 concluded that the two clusters are at the same distance. A distance of $2.5 \mathrm{kpc}$ is adopted for our study. The Carina Nebula is known to contain the largest number of early-type stars in the Milky Way, with a total of 64 O-type stars (Feinstein 1995). In particular, Tr 14 and $\operatorname{Tr} 16$ include six exceedingly rare main-sequence $\mathrm{O} 3$ stars. The presence of these very massive stars indicates that the two clusters are extremely young. Tr 16 contains a Wolf-Rayet star, HD 93162, and is also the host cluster to the famous luminous blue variable (LBV), $\eta$ Carinae, which is arguably the most massive star in our Galaxy (MJ93). With such a plethora of unusually massive 
This is an unedited preprint of an article accepted for publication in The Astrophysical Journal. The final published article may differ from this preprint. Copyright 2006 by The American Astronomical Society. Please cite as 'ApJ preprint doi:10.1086/'510184".

stars, the Carina Nebula is a unique laboratory to study not only the massive star formation process, but also the interplay among massive stars, interstellar media and low-mass star formation.

Of the existing methods to identify young stellar populations, the use of X-ray emission is perhaps the least biased (Feigelson et al. 2002). Indeed, X-ray surveys have in recent years been very successful in identifying the pre-main sequence (PMS) population in star-forming regions, from deeply embedded Class I young stellar objects (YSOs), low-mass PMS stars of T Tauri types, intermediate-mass PMS stars of Herbig Ae/Be types, to zero-age mainsequence stars. For main-sequence stars, from late A to M dwarfs, X-rays are believed to originate from the hot coronal gas, heated by stellar dynamo magnetic fields (Maggio et al. 1987). For late type PMS stars, i.e., classical T Tauri stars (CTTSs) and weak-lined T Tauri stars (WTTSs), the X-ray emission is also attributed to solar-like coronal activity (Feigelson \& DeCampli 1981; Walter \& Kuhi 1984) but elevated by a factor of 10-104 (Walter \& Barry 1991; Feigelson \& Montmerle 1999). X-ray observations are especially effective in identification of WTTSs, which lack the other signatures of PMS stars such as the infrared excess or strong $\mathrm{H}_{\alpha}$ emission lines.

For massive $\mathrm{O}$ and early $\mathrm{B}$ type stars, the most widely accepted X-ray emission model is the wind-shock model (Lucy 1982), in which the X-rays are produced in shocks arising from hydrodynamical instabilities in the radiatively driven strong stellar winds. Since the X-ray emission from hot stars is mostly soft and nearly constant, it is attributed to small-scale wind shocks (Lucy \& White 1980; Owocki \& Cohen 1999). Recent X-ray observations of some hot stars, however, cannot be explained by the classical wind-shock model. For example, Feigelson et al. (2002) discovered rapid variability in an O9.5 star and several early-B stars in the Trapezium. As another example, Stelzer et al. (2005) found the majority of the O to early-B stars in the Orion Trapezium to show a combination of soft emission, hard-spectrum short-lived flares and/or rotational modulation. Some of these X-ray properties not typical of hot stars can be attributed to confinement of the wind by a strong magnetic field, resulting in large-scale shocks (Babel \& Montmerle 1997; ud-Doula \& Owocki 2002; Schulz et al. 2003). This scenario is further supported by independent indicators of magnetic fields on hot stars, such as cyclic variability of wind properties (Kaper et al. 1999; de Jong et al. 2001) and the existence of nonthermal radio emission (Abbott et al. 1984; Van Loo et al. 2004).

Three observations of the Carina Nebula have been made by the Chandra X-ray Observatory in September 1999, namely, obsIDs=50, 51 and 1249, placing $\eta$ Carinae at the focus of the I3 chip of the ACIS-I detector. The first two sets of observations obsIDs=50 and 1249 were made in the timed exposure mode, whereas the third set obsID=51 was made in the continuous clocking mode. Seward et al. (2001) reported the observations of $\eta$ Carinae using 
This is an unedited preprint of an article accepted for publication in The Astrophysical Journal. The final published article may differ from this preprint. Copyright 2006 by The American Astronomical Society. Please cite as 'ApJ preprint doi:10.1086/'510184".

two observations (obsIDs=1249 and 51). Using the single observation obsID=1249, Evans et al. (2003, hereafter E03) presented the luminosities and hardness ratios of the hot stars in the clusters $\operatorname{Tr} 16$, and $\operatorname{Tr} 14$. The low-resolution spectra of luminous sources were discussed by Evans et al. (2004). In the present paper, we combined the two Chandra observations of obsIDs 1249 and 50, which were observed in timed exposure mode. By merging the two observations, we gained better sensitivity than E03, and detected more than twice the number of sources reported by E03. The Chandra data were complemented by deep NIR $(J, H$, and $K_{s}$ ) imaging observations, carried out at the Infrared Survey Facility (IRSF) 1.4 m telescope, to better characterize the nature of the stellar X-ray sources. Our analysis resulted in some 38 X-ray emitting known OB stars and about 300 PMS candidates. Moreover, on the basis of NIR and X-ray properties of the known OB stars in the region, we discovered 16 massive star candidates. These massive star candidates probably escaped previous detection because of their large extinction in the optical wavelengths. Our study has produced the most comprehensive young star sample so far of $\operatorname{Tr} 14$ and $\operatorname{Tr} 16$, thus allowing us to delineate the star formation history in this seemingly devastating environment. In particular, we report the discovery of an embedded ( $A_{\mathrm{V}} \sim 15 \mathrm{mag}$ ) young stellar group, located to the south-east of $\operatorname{Tr} 16$ and sandwiched between two dense molecular clouds. Similar patterns of newly formed stars in relation to clouds seem to encompass the Carina Nebula, a manifestation of triggered star formation via the collect-and-collapse process (Deharveng, Zavagno \& Caplan 2005).

The rest of this paper is organized as follows. $§ 2$ describes the Chandra and the IRSF observations and the data analysis. In $\S 3$, we present the cross-identification of Chandra sources with the optical spectroscopic information available in the literature and with our NIR sample. We discuss the results and implication of our study in $\S 4$, and summarize the results in $\S 5$.

\section{OBSERVATIONS AND DATA REDUCTION}

\subsection{X-ray data - Chandra}

The Carina Nebula has been observed by the Chandra X-ray Observatory with the ACIS-I three times. All three observations (obsID=50, 51, and 1249) were made in 1999 September, shortly after the launch. The observation made in the continuous clocking mode $($ obsID $=51)$ does not have the spatial resolution in one dimension and is thus not useful for our work. The other two ACIS-I observations are used in this work and their parameters are listed in Table 1. 
This is an unedited preprint of an article accepted for publication in The Astrophysical Journal. The final published article may differ from this preprint. Copyright 2006 by The American Astronomical Society. Please cite as 'ApJ preprint doi:10.1086/'510184".

We began our data analysis with a Level 1 processed event file. Cosmic-ray afterglows, hot pixels, and events with poor grades were filtered out. The filtering process was done using the Chandra Interactive Analysis of Observations (CIAO) package, following the Science Threads from the Chandra X-Ray Center. We further removed the periods with a high background. The total useful exposure time from the two observations combined was $18.1 \mathrm{ks}$. The charge transfer inefficiency (CTI) and time-dependent gain corrections were not applied, because the focal plane temperature of these two observations was not the nominal $-120 \mathrm{C}$.

The two observations were merged into one image and the sources are shown in Figure 1. The extraction of source counts was carried out for the two observations separately. An energy range of $0.4-6.0 \mathrm{keV}$ was adopted in the analysis to optimize the detection of PMS stars and reduce the number of spurious detections. The CIAO program WAVDETECT was utilized to detect sources in the merged image. We ran wavelet scales ranging from 1 to 16 pixels in steps of $\sqrt{2}$ with a source significance threshold of $3 \times 10^{-6}$. Spurious detections, e.g., in the partial shell of X-ray emission surrounding $\eta$ Carinae (Seward et al. 2001) and along the read-out trail due to $\eta$ Carinae itself, were identified and removed. At the end, we obtained 454 valid sources. For comparison, the lower spatial resolution XMM-Newton observations of the Carina Nebula detected only 80 X-ray sources for the energy range of 0.3-12.0 keV (Albacete Colombo et al. 2003).

To measure the X-ray counts from each source, we used a circular source region and an annular background region, both centered on the position given by WAVDETECT. The radius of the source region was $R(95 \% \mathrm{EE})$, the radius that encircles $95 \%$ of the energy (Feigelson et al. 2002), and the radii of the background region were 1.2 and $1.5 R(95 \% \mathrm{EE})$. Sources that fell within the background regions were excised. We utilized the CIAO DMEXTRACT tool to extract the source and background counts for each of the two observations. The background counts scaled to the source extraction area were subtracted from the source counts. The total counts of each source were then computed by combining the two observations and the count rates for a total exposure time of 18,120 s were calculated.

The source counts extracted by us from the single observation ID $=1249$ were generally consistent (within 10\%) with those reported by E03 for the same dataset, except for a handful of cases. The three largest discrepancies are in the measurement of HD 93129 A/B (O3 I) located near the edge of the Chandra image, HD 93160 (O6.5 V), and HDE 303311 (O5). There are two possibilities for the discrepancy. First, our energy band, 0.4-6.0 keV, is broader than E03's 0.5-2.04 keV, so our measurements of a hard source would appear brighter. Second, we use an annular background aperture around each source, while E03 used two large off-source regions to estimate the background. Our background estimate should thus be more accurate, especially for stars surrounded by diffuse emission. Both 
This is an unedited preprint of an article accepted for publication in The Astrophysical Journal. The final published article may differ from this preprint. Copyright 2006 by The American Astronomical Society. Please cite as 'ApJ preprint doi:10.1086/'510184".

HD 93129 A/B and HD 93160 are associated with diffuse emission and hence the stellar fluxes must have been overestimated by E03. HD 93129 A/B is located near the edge of the ACIS-I image, and the large point-spread-function also contributes to the uncertainty in the source count extraction.

\subsection{Near-Infrared Data - IRSF}

We carried out near-infrared imaging observations toward the Carina Nebula using the SIRIUS (Simultaneous InfraRed Imager for Unbiased Survey) camera mounted on the IRSF $1.4 \mathrm{~m}$ telescope in Sutherland, South Africa. The SIRIUS camera (Nagayama et al. 2003) is equipped with three HAWAII arrays of $1024 \times 1024$ pixels and provides simultaneous observations in the three bands, $J(1.25 \mu \mathrm{m}), H(1.63 \mu \mathrm{m})$ and $K_{s}(2.14 \mu \mathrm{m})$ using dichroic mirrors. Each image has a field of view of $7 ! 8 \times 7 ! 8$ with a plate scale of $0{ }^{\prime \prime} 45$ pixel $^{-1}$. In April 2003, nine pointings were observed effectively covering $22^{\prime} \times 22^{\prime}$ which encompassed the Chandra field. Figure 2 shows the NIR color-composite image of the Nebula $\left(\sim 22^{\prime} \times 22^{\prime}\right)$, centered on $\eta$ Carinae. Tr 16 can be seen near the center of the image, whereas $\operatorname{Tr} 14$ is in the northwest (the top right corner) of the image. The central coordinates of the mapped area are R.A. $=10^{h} 45^{m} 05^{s}$ and Dec. $=-59^{\circ} 38^{\prime} 52^{\prime \prime}$. For each pointing, 30 dithered frames were observed, each with an integration time of $30 \mathrm{~s}$, giving a total integration time of $900 \mathrm{~s}$. Two pointings (\#5 and \#6) of the April 2003 observations were affected by weather fluctuations, so were re-observed in January 2005. For the 2005 observations, 45 dithered frames were observed, each with an integration time of $20 \mathrm{~s}$, yielding a total integration time of $900 \mathrm{~s}$ for each pointing. The typical seeing during our observations ranged from $1^{\prime \prime}$. 0 to $1^{\prime \prime} .4$ and the airmass ranged from 1.2 to 1.5. The standard stars No. 9144 and 9146 from Persson et al. (1998) were observed for photometric calibration.

We used the NOAO's IRAF (Image Reduction and Analysis Facility) package to reduce the IRSF data. The standard procedures for image reduction were applied, including dark current subtraction, sky subtraction and flat field correction. The images in each band were then combined for each pointing to achieve a higher signal-to-noise ratio. We performed photometry on the reduced images using IRAF's DAOPHOT package (Stetson 1987). Since the field is crowded, we performed PSF (point spread function) photometry in order to avoid source confusion. To construct the PSF for a given image, we chose about 15 bright stars well isolated from neighboring stars and located away from nebulosities or the edges of the image. The task ALLSTAR of DAOPHOT was then used to apply the average PSF of the 15 stars to all the stars in the image. The instrumental magnitude of each star was then derived. The instrumental magnitudes were then calibrated against the standard stars observed on 
This is an unedited preprint of an article accepted for publication in The Astrophysical Journal. The final published article may differ from this preprint. Copyright 2006 by The American Astronomical Society. Please cite as 'ApJ preprint doi:10.1086/'510184".

each night.

\section{X-RAY SOURCES AND STELLAR COUNTERPARTS}

Optical spectroscopic observations of the stars in $\operatorname{Tr} 14$ and $\operatorname{Tr} 16$ have been carried out by several groups, e.g., Walborn (1973), Walborn (1982), Levato et al. (1981), and FitzGerald \& Mehta (1987). The latest work by MJ93 lists the brightest and bluest stars of the two clusters (Table 4 in MJ93), as well as some new spectral types (Table 5 in MJ93). We have used these lists to find X-ray counterparts. With a search radius of $1^{\prime \prime}$ for sources within a $2^{\prime}$ off-axis angle, $2^{\prime \prime}$ for those with a $2-5^{\prime}$ off-axis angle, and $3^{\prime \prime}$ for those with an off-axis angle larger than $5^{\prime}$, our cross-identification resulted in 30 OB stars as counterparts of our X-ray sources. Apart from MJ93, we also searched for possible counterparts using SIMBAD ${ }^{1}$ with the same search radius criteria as above. The reliability of any photometric or spectroscopic information obtained from SIMBAD was confirmed by looking into the original literature. Our search using SIMBAD resulted in additional finding of 8 OB stars in the region, for all of which the spectral types were estimated by Tapia et al. (1988) by the photometric Q method (Johnson \& Morgan 1953). A visual inspection of images indicated that none of the known OB stars has more than one X-ray source within the search radius, so the cross-identification should be secure. The average offset between the optical and X-ray positions is $\sim 0.7^{\prime \prime}$.

We also used our NIR data to search for X-ray counterparts. With the same search radius criteria as described in the previous paragraph, we found NIR counterparts for 425 sources. Thus, $94 \%$ of the X-ray sources were found to have NIR counterparts. There are 50 cases where we found multiple (in all cases two) NIR counterparts, 33 of which are with off-axis angles smaller than $5^{\prime}$. Since the astrometric accuracy of Chandra is sufficiently good for on-axis sources, we considered the closest NIR source to be the counterpart in these cases. A visual inspection of each of these cases also showed that the cross-identification is straightforward. For the rest 17 cases with off-axis angles larger than $5^{\prime}$, it is difficult to ascertain which NIR source is the real counterpart given the relatively large positional uncertainties of Chandra for off-axis sources. In these cases, we considered the brightest NIR source to be the X-ray counterpart, although this may not be true in all the cases.

Among our NIR counterparts of the X-ray sources, for 51 cases out of 425, the NIR photometric errors are larger than $0.1 \mathrm{mag}$ in one or more bands. Most of these large photometric errors are associated with stars around $\operatorname{Tr} 16$, for which the quality of the NIR photometry is compromised because of a large number of bright stars and the nebulosity

\footnotetext{
${ }^{1}$ http://simbad.u-strasbg.fr/sim-fid.pl
} 
This is an unedited preprint of an article accepted for publication in The Astrophysical Journal. The final published article may differ from this preprint. Copyright 2006 by The American Astronomical Society. Please cite as 'ApJ preprint doi:10.1086/'510184".

around $\eta$ Carinae. Since we are using the NIR colors of the sources to diagnose their young stellar nature, an uncertainty larger than $0.1 \mathrm{mag}$ would defeat the purpose. Hence, in our analysis we consider only those cases for which the photometric uncertainties are less than $0.1 \mathrm{mag}$ in all three bands. This leaves us with 374 sources. For our analysis, we have converted the NIR photometry into the California Institute of Technology (CIT) system using the color transformations between the IRSF and CIT systems as given in Nakajima et al. (2005).

\section{RESULTS AND DISCUSSION}

Figure 3 shows the NIR color-color diagram of all the 374 X-ray sources with NIR photometric errors less than $0.1 \mathrm{mag}$. The solid curve represents the dwarf and giant loci (Bessell \& Brett 1988). The parallel dotted lines represent the reddening vectors, with $A_{J} / A_{V}=0.282, A_{H} / A_{V}=0.272$, and $A_{K} / A_{V}=0.112$ (Rieke \& Lebofsky 1985). The dashed line indicates the locus for dereddened CTTSs (Meyer et al. 1997). As can be seen, the NIR colors of the X-ray sources in our sample, in addition to massive stars, show a large population of $\mathrm{T}$ Tauri type candidates. The different symbols in the figure indicate the following: (a) X-ray sources cross-identified with the known massive stars of O or B types are shown as filled circles; (b) Some 16 X-ray bright sources found with NIR colors characteristic of massive stars, henceforth referred to as candidate OB stars, are shown as diamonds; (c) A compact, partially embedded stellar group of $10 \mathrm{X}$-ray sources is found coinciding with the dust lane to the south-east of $\eta$ Carinae and behind the ionization fronts. The stars of this group are shown as triangles; (d) All other X-ray sources that do not fall into any of the above categories are shown as dots. Figure 4 shows the NIR color-magnitude diagram of all these sources. The solid line in the figure represents the unreddened main sequence (Koornneef 1983) at $2.5 \mathrm{kpc}$ and the dashed line shows the reddening vector for $A_{V}$ equal to $10 \mathrm{mag}$. In the following subsections, we discuss the known OB stars, the candidate OB stars, the CTTS candidates and the embedded stellar group in detail.

\subsection{Known OB stars}

The X-ray sources cross-identified with known OB stars are presented in Table 2. Columns (1) and (2) give the coordinates of each X-ray source, and column (3), the identifier of its optical counterpart. Columns (4)-(6) list the optical $B$ and $V$ magnitudes and spectral type, in most cases adopted from MJ93 or otherwise from Tapia et al. (1988). Column (7) lists the color excess, $E(B-V)$, derived by MJ93 from photometric and spectroscopic 
This is an unedited preprint of an article accepted for publication in The Astrophysical Journal. The final published article may differ from this preprint. Copyright 2006 by The American Astronomical Society. Please cite as 'ApJ preprint doi:10.1086/'510184".

observations; the latter were used to estimate the intrinsic stellar $E(B-V)_{0}$ (FitzGerald 1970). Column (8) shows the bolometric magnitude, $M_{\mathrm{bol}}$, taken from Massey et al. (2001). Note that for a small number of cases where the spectral types were taken from Tapia et al. (1988), the color excesses and bolometric magnitudes were estimated using their spectral types. Columns (9)-(11) list the IRSF $J, H$ and $K_{s}$ magnitudes. Column (12) lists the background-subtracted X-ray counts of the source as derived by the DMEXTRACT tool of the CIAO software, described in $§ 2.1$, from which the unabsorbed flux was derived using the WebPIMMS ${ }^{2}$ interface. To convert the X-ray count rate to the flux, the Raymond Smith Plasma model with a temperature $\log T=6.65$, equivalent to $0.384 \mathrm{keV}$, typically found in young associations (E03), was adopted. We found that using a $\log T$ between 6.4 to 7.1 would make a difference of a factor of two or less in the derived X-ray luminosities. For the extinction correction, the color excess $E(B-V)$ of each source was used to estimate the absorbing column density, $N_{H}$, by adopting $N_{H} / E(B-V)=5.8 \times 10^{21}$ atoms $\mathrm{mag}^{-1} \mathrm{~cm}^{-2}$ (Bohlin et al. 1978). Column (13) gives the unabsorbed X-ray flux, and column (14), the X-ray luminosity, computed by adopting a distance of $2.5 \mathrm{kpc}$. Column (15) contains the logarithmic ratio of the X-ray luminosity to the stellar bolometric luminosity derived from the bolometric magnitude, i.e., column (8). The last two columns, (16) and (17), list the angular separation $(r)$ between the X-ray source and its optical counterpart, and the off-axis angle $(\theta)$ of the $\mathrm{X}$-ray source in the Chandra image, respectively.

All but two O stars from MJ93 were found to be X-ray emitters. The two O stars with no X-ray counterpart, HD 93128 (an O3V star) and Tr 14-9 (an O8V star) are both located near the edge in the Chandra image, giving a plausible explanation for their non-detection. HD 93128 was indeed detected in the XMM-Newton data (Albacete Colombo et al. 2003). Two more O stars CD 583529 (an O6.5V star) and Tr 16-200 (an O9V star) which are also near the edge in the Chandra image, were not detected by E03, but were detected in our data. The star CD 583529 is very bright, 350 counts in our analysis (see Table 2) but E03 derived only an upper limit of $\sim 30$ counts. Possible reasons to account for its nondetection by E03 may be its location in the crowded Tr 14 region and association with pronounced diffuse X-ray emission.

While X-ray emission is detected from every known O star in the Chandra field (barring the two stars on the edge), only 4 out of 38 (or about 10\%) of the B stars (early-B) from MJ93 are detected with X-ray emission. For most of these nondetected early B stars, the upper limits are lower than the X-ray luminosities of detected known OB stars, implying that these $\mathrm{B}$ stars have weaker X-ray emission. In our data, however, there are 3 B3-type, 3 B5-type

\footnotetext{
${ }^{2}$ http://heasarc.gsfc.nasa.gov/Tools/w3pimms.html
} 
This is an unedited preprint of an article accepted for publication in The Astrophysical Journal. The final published article may differ from this preprint. Copyright 2006 by The American Astronomical Society. Please cite as 'ApJ preprint doi:10.1086/'510184".

and 1 B7-type stars found associated with X-ray emission. Mid- to late-B type stars are supposed to be X-ray quiet as they have neither strong enough stellar winds, as in the case of $\mathrm{O}$ or early $\mathrm{B}$ stars, nor convective zones to power the chromospheric/coronal activities, as in the case of late-type stars. However, mid- to late-B type stars have been found to be X-ray emitters in earlier studies, e.g., by Cohen et al. (1997). The X-ray luminosities of the mid- to late-type B stars in our sample range from $10^{30}$ to $10^{31} \mathrm{ergs} \mathrm{s}^{-1}$, comparable to those of the $\mathrm{T}$ Tauri candidates in the same sample, providing circumstantial evidence for T Tauri or Herbig Ae/Be companions to account for the X-ray emission. Nonetheless the possibility of a so far unknown emission mechanism intrinsic to mid- to late-B stars cannot be ruled out.

Figure 5 shows the distribution of the X-ray luminosities of the detected known OB stars (unshaded) in our field. The X-ray luminosities of the known OB stars range from $\sim 10^{30}$ to $10^{34} \mathrm{ergs} \mathrm{s}^{-1}$, with the Wolf-Rayet star (HD 93162) being the brightest X-ray source in the sample, with $\log L_{\mathrm{X}}=34.12 \mathrm{ergs} \mathrm{s}^{-1}$. This star is known to be unusually bright in X-rays compared to other W-R stars in the region (E03). Strong X-ray emission may be produced by the colliding stellar winds between binary components of massive stars. Although HD 93162 has been thought to be a single star, a recent W-R catalog by van der Hucht (2001) suggested that it might be a binary on the basis of diluted emission lines (see the discussion in E03).

The X-ray luminosities of $\mathrm{OB}$ stars are known to be related to the stellar bolometric luminosities, namely, $L_{\mathrm{X}} \propto 10^{-7} L_{\text {bol }}$ (Chlebowski et al. 1989; Kudritzki et al. 1996). This linear scaling of $L_{\mathrm{X}}$ with $L_{\mathrm{bol}}$ can be explained by the wind-shock X-ray emission model in hot stars (Owocki \& Cohen 1999). In our sample, most stars have $\log \left(L_{\mathrm{X}} / L_{\mathrm{bol}}\right)$ between -6 to -7 (Fig. 6). In the figure the outliers are labeled by their spectral types. The star $\eta$ Carinae has a $\log \left(L_{\mathrm{X}} / L_{\mathrm{bol}}\right)$ equal to -7.5 . The spectra of this peculiar star have been discussed by several groups, e.g., Chlebowski et al. (1984), Corcoran et al. (1995), Ishibashi et al. (1999), Seward et al. (2001), and Albacete Colombo et al. (2003). The Chandra observations of $\eta$ Carinae resolved the central source from the surrounding partial shell or ring (Seward et al. 2001, see Fig. 1). At energies lower than $1 \mathrm{keV}$ only the ring can be seen, whereas the central source has emission at energies even higher than $7 \mathrm{keV}$. The central source therefore has a hard X-ray spectrum and is strongly absorbed, whereas the shell has soft emission (Seward et al. 2001). The X-ray luminosity of the central source before absorption by the surrounding material and interstellar medium was estimated to be $\log L_{\mathrm{X}} \sim 34.7 \mathrm{ergs} \mathrm{s}^{-1}$ by Seward et al. (2001) in the energy range $0.2-10 \mathrm{keV}$, whereas our measurement of the central source is $\log L_{\mathrm{X}} \sim 32.7 \mathrm{ergs} \mathrm{s}^{-1}$ in the $0.4-6.0 \mathrm{keV}$ energy range (see Table 2). The XMM-Newton observations also found a hard X-ray core and a non-uniform elliptical shell with soft emission (Albacete Colombo et al. 2003, see their Fig. 6). They measured the 
This is an unedited preprint of an article accepted for publication in The Astrophysical Journal. The final published article may differ from this preprint. Copyright 2006 by The American Astronomical Society. Please cite as 'ApJ preprint doi:10.1086/'510184".

X-ray luminosity to be $\log L_{\mathrm{X}} \sim 36 \mathrm{ergs} \mathrm{s}^{-1}$ in the range $0.3-12 \mathrm{keV}$ (Albacete Colombo et al. 2003, their source No. 50), which also includes soft emission from the shell, unlike the Chandra X-ray luminosity by Seward et al. (2001), which measures only the hard spectrum core. Along with $\eta$ Carinae, the W-R star, HD 93162, and an O8.5V star, Tr 16-22, also deviate from the mean $L_{\mathrm{X}} / L_{\text {bol }}$ value found for our sample of known OB stars. These stars show high $L_{\mathrm{X}} / L_{\text {bol }}$ values, with $\log \left(L_{\mathrm{X}} / L_{\mathrm{bol}}\right)=-5.39$ and -5.32 , respectively. Along with the W-R star HD 93162, Tr 16-22 is also among the brightest X-ray sources, with $\log L_{\mathrm{X}}=32.83 \mathrm{ergs} \mathrm{s}^{-1}$, brighter by a factor of 5-20 compared to other O8.5V stars and even brighter than two of the five O3 stars (HDE 303308, and Tr 16-244) in our sample. HD 93162 and $\operatorname{Tr} 16-22$ are among the hardest X-ray sources in the sample of known OB stars (E03), and also among the brightest XMM-Newton sources (Albacete Colombo et al. 2003), with $\log L_{\mathrm{X}}=34.26 \mathrm{ergs} \mathrm{s}^{-1}$ and $33.30 \mathrm{ergs} \mathrm{s}^{-1}$, respectively. The XMM-Newton observations include harder X-ray emission (0.3-12 keV) than in our Chandra data, so the higher XMM-Newton luminosities indicate that HD 93162 and Tr 16-22 indeed have hard X-ray spectra, indicating the existence of colliding stellar winds (Corcoran 2003).

The other X-ray sources that do not satisfy the $L_{\mathrm{X}} / L_{\mathrm{bol}}$ relation are mid-B or late-B stars. A study by Berghöfer et al. (1997) about the X-ray properties of OB stars using the $R O S A T$ database showed that the $L_{\mathrm{x}} / L_{\mathrm{bol}}$ relation extends to as late as the spectral type B1.5. In our sample, there are 3 B3 stars which seem to satisfy this relation, and all the stars later than B3 deviate significantly from the mean value of $L_{\mathrm{X}} / L_{\mathrm{bol}}$ for $\mathrm{O}$ and early-B stars. Some recent studies, e.g., Stelzer et al. (2005) and Townsley et al. (2006) have found, however, several cases where $L_{\mathrm{X}} / L_{\mathrm{bol}}$ is different from $10^{-7}$. Stelzer et al. (2005) found that out of 9 O to early-B (up to B4) stars in the Orion Trapezium, about half of them show $\log \left(L_{\mathrm{X}} / L_{\mathrm{bol}}\right)$ between -6 and -7 , with the total range of the sample being -5 to -8 .

We checked for variability in the X-ray brightness of known OB stars using the counts from the two observations ( $\mathrm{obsIDs}=50$ and 1249). To determine whether a source is variable or not, we computed the mean percentage variation between the count rates of the sources detected from the two observations, obsIDs 1249 and 50. We defined this mean percentage variation as a measure of the systematic fluctuations, $\sigma$, and compared the difference in the count rates of each X-ray source between the two events files (obsID=1249 and 50) with respect to $\sigma$. A difference larger than $3 \sigma$ was considered as an X-ray variable. None of the bright X-ray sources (i.e., with counts greater than 100 in at least one of the two observations) are variable. Among the fainter sources (with counts $\sim 15-30$ in at least one observation), there are four cases that showed larger than $3 \sigma$ variations, namely, $\operatorname{Tr} 14-18$ (B0V type), Tr 16-11 (B1.5 type), CPD -59 2616 (B3 type) and Tr 16-200 (O9V type). The color excesses of these sources $E(B-V)$ range from 0.43 to 0.60 , close to the mean value of $E(B-V)=0.52$ for the known OB stars. The hardness ratios of these faint sources are in 
This is an unedited preprint of an article accepted for publication in The Astrophysical Journal. The final published article may differ from this preprint. Copyright 2006 by The American Astronomical Society. Please cite as 'ApJ preprint doi:10.1086/'510184".

general too uncertain to see if the X-ray variables have different hardness ratios compared to other sources which appear to have steady X-ray emission. Albacete Colombo et al. (2003) carried out a time variability study of the X-ray sources with the XMM-Newton data. They found seven sources to be variable, out of which only one is an O type star (HD 93205, spectral type O3V), which is also a known binary (E03). In our analysis, however, this star did not appear to be varying. It is interesting to note that, while a few B stars with low counts (a few to a few tens) seem to vary - we will not discuss them further because the count statistics is very poor to decide if they are truly variable - none of the O stars (except for the O9 star Tr 16-200 discussed above), even with poor counts, vary by more than $3 \sigma$ between the two observations.

\subsection{Candidate OB stars}

There are 16 anonymous stars in our sample which have NIR and X-ray properties similar to those of the known OB stars in the region. On the basis of their NIR colors and brightness $\left(K_{s}<12 \mathrm{mag}\right)$, these stars appear to be massive stars of $\mathrm{O}$ or B type, but we could not find their spectral type information in the literature (e.g., in MJ93 or SIMBAD). The optical and NIR magnitudes of these candidate OB stars, along with their X-ray counts, $\mathrm{X}$-ray flux and X-ray luminosities, are listed in Table 3. To determine their X-ray fluxes from the counts, we made use of WebPIMMS. For the extinction correction, we used an average $E(B-V)=0.52$ (MJ93), as we did not have the spectral class information to determine their individual color excesses. Other parameters used to convert to X-ray fluxes from the X-ray counts remained the same as for the known OB stars. For the known OB stars, where we used individual $E(B-V)$ values for the extinction correction, use of an average $E(B-V)$ would make a difference of a factor of two or less in the derived X-ray luminosities.

As can be seen in Figure 3, the candidate OB stars (diamonds) are either as red as or redder than the known OB stars. They have a similar NIR brightness as the known OB stars, with two of them being even brighter than $K_{s}=8 \mathrm{mag}$ (see Fig. 4 ). In the optical wavelengths, these candidate $\mathrm{OB}$ stars are fainter and redder than the known OB stars (Figure 7) - indicative of significant dust extinction — while both samples show a comparable range of X-ray luminosities (see Figure 5). Thus, from the NIR and X-ray properties, it appears that these stars have escaped earlier optical spectroscopic studies because of their large optical extinction. The addition of these massive stars expands substantially the list of known luminous stars and hence contributes significantly to the stellar energy budget of the region. 
This is an unedited preprint of an article accepted for publication in The Astrophysical Journal. The final published article may differ from this preprint. Copyright 2006 by The American Astronomical Society. Please cite as 'ApJ preprint doi:10.1086/'510184".

\subsection{PMS candidates}

Although much work has been done on the massive stellar contents in $\operatorname{Tr} 14$ and $\operatorname{Tr} 16$, the T Tauri population in the region has not been well studied. Tapia et al. (2003) presented $U B V R I J H K$ photometry of Tr 14 and Tr 16, noting that some stars have NIR excesses, and estimated the age of these two clusters to be 1-6 million years. By using the criteria of NIR colors given by Meyer et al. (1997), we identified about 180 X-ray stars as CTTS candidates. The NIR colors also suggest quite a number of possible WTTS candidates. The X-ray and NIR data together hence reveal, in addition to massive stars, a large population of low-mass PMS candidates. The distribution of X-ray luminosities of the CTTS candidates is shown in Figure 8. In comparison with Figure 5, one can see that the X-ray luminosities of the $\mathrm{T}$ Tauri candidates are on the average lower than those of massive stars. This is consistent with the notion that late-type stars have weaker X-ray emission. To the best of our knowledge, our sample represents the most comprehensive sample so far of the young stellar population in $\operatorname{Tr} 14$ and $\operatorname{Tr} 16$. A detailed analysis of this sample will be presented elsewhere.

\subsection{A compact embedded X-ray stellar group}

Megeath et al. (1996) found a deeply embedded IRAS object IRAS 10430-5931 associated with a bright-rimmed globule with a mass of $\sim 67 M_{\odot}$ located to the south of $\operatorname{Tr} 16$. They also found several sources with NIR excesses around this IRAS object, providing the first indication of ongoing star-forming activity in the Carina region. Later, a mid-infrared study by Smith et al. (2000) discovered several clumps along the edge of the dark cloud east of $\eta$ Carinae, including a clump associated with IRAS 10430-5931. They noted that each of these clumps is a potential site for triggered star formation, judging from their peripheral locations behind the ionization fronts relative to the Nebula.

We found a group of $10 \mathrm{X}$-ray sources concentrated in a field of $2^{\prime} \times 4^{\prime}$, spatially close $\left(\sim 4^{\prime}\right)$ to IRAS 10430 and coincident with a prominent dark V-shaped dust lane bisecting the Carina Nebula. Adopting a distance of $2.5 \mathrm{kpc}$, the physical size of this star group is about 2 pc. Each of these 10 sources has an NIR counterpart, which is listed in Table 4 with their X-ray coordinates, $J, H$, and $K_{s}$ magnitudes, X-ray counts and $A_{\mathrm{V}}$. We use the star identification number in column 1 of Table 4 in further discussion. Stars in this group are bright (two of them are OB star candidates) and suffer a large amount of reddening, as seen in the NIR color-color and color-magnitude diagrams (the triangles in Figures 3 and 4). The brightest sources (stars 5, 7 and 8) in NIR, with $K_{s} \sim 8.5-10.5$ mag, are also relatively bright in X-rays, with $L_{\mathrm{X}} \sim 10^{32} \mathrm{ergs} \mathrm{s}^{-1}$. Star 5 is a known O4 star (Smith et al. 1987). Our NIR magnitudes for this star match those reported by Smith et al. (1987). 
This is an unedited preprint of an article accepted for publication in The Astrophysical Journal. The final published article may differ from this preprint. Copyright 2006 by The American Astronomical Society. Please cite as 'ApJ preprint doi:10.1086/'510184".

Except for star 5, we could not find any optical photometric or spectroscopic information in the literature for the others sources. The bright NIR and X-ray stars 5, 7, and 8 are visible in the DSS (Digitized Sky Survey) image, whereas the other sources, most of which show a large extinction in NIR are indeed not discernible at all in the DSS image. Figure 9 shows the IRSF $K_{s}$ image with the sources marked. Stars 1, 6, 9 and 10 are peculiar because they are highly extincted $\left(A_{V} \sim 10-15 \mathrm{mag}\right.$ as estimated from their NIR colors), yet are X-ray bright with $L_{\mathrm{X}} \sim 10^{33} \mathrm{ergs} \mathrm{s}^{-1}$. What could be the nature of these sources? Their NIR fluxes and colors, along with their non-detection in the DSS image, imply that they could be reddened T Tauri or class I objects. However their X-ray luminosities are much higher than observed in typical $\mathrm{T}$ Tauri stars $\left(<10^{32} \mathrm{ergs} \mathrm{s}^{-1}\right)$. One possibility is that they are heavily embedded massive stars. The rest of the sources in the group, stars 2, 3 and 4 are relatively faint in both NIR and X-rays, thus appear to be reddened T Tauri stars.

Figure 10 shows all $454 \mathrm{X}$-ray sources overlaid on the mosaic $K_{s}$ image centered around $\eta$ Carinae, with the contours showing ${ }^{12} \mathrm{CO}(1-0)$ emission (Brooks et al. 1998). The group of young stars discussed above (shown as crosses) is seen in the bottom left 'sandwiched' between two clouds. The known OB stars are marked by boxes, whereas the candidate OB stars are indicated by diamonds and all other X-ray sources by circles. One sees immediately the general paucity of young stars with respect to molecular clouds. Tr 16 itself (seen near the center in the figure) is also "sandwiched" between the north-west and south-east cloud complexes. Likewise, all the X-ray sources (i.e., young stars) associated with these clouds in turn are seen either intervening between clouds or situated near the cloud surfaces facing Tr 16. The morphology of young stellar groups and molecular clouds peripheral to an H II region (i.e., Tr 16 here) fits closely the description of the collect-and-collapse mechanism for massive star formation first proposed by Elmegreen \& Lada (1977), and recently demonstrated observationally by Deharveng, Zavagno \& Caplan (2005) and Zavagno et al. (2006). The expanding ionization fronts from an H II region compress the outer layer of a nearby cloud so that the gas and dust accumulate until the critical density is reached for gravitational collapse to form next-generation stars, subsequently casting out their own cavities. This collect-collapse-clear process may continue as long as massive stars are produced in the sequence and there is sufficient cloud material in the vicinity.

\subsection{The X-ray luminosity function of $\operatorname{Tr} 16$}

Feigelson \& Getman (2005) pointed out that the X-ray luminosity functions (XLFs) of young stellar clusters show two remarkable characteristics. First, the shapes of the XLFs of different young stellar clusters are very similar to each other once the tail of the 
This is an unedited preprint of an article accepted for publication in The Astrophysical Journal. The final published article may differ from this preprint. Copyright 2006 by The American Astronomical Society. Please cite as 'ApJ preprint doi:10.1086/'510184".

high-luminosity $\mathrm{O}$ stars $\left(\log L_{\mathrm{X}}>31.5 \mathrm{ergs} \mathrm{s}^{-1}\right)$ is omitted. Secondly, the shape of this 'universal' XLF in the $0.5-8.0 \mathrm{keV}$ energy range resembles a lognormal distribution with a mean $\log L_{\mathrm{X}} \approx 29.5 \mathrm{ergs} \mathrm{s}^{-1}$ and a standard deviation $\sigma\left(\log L_{\mathrm{X}}\right) \approx 0.9$ (see Figure 2 in Feigelson \& Getman 2005). The Chandra observations used in this work include only part of $\operatorname{Tr} 14$. For Tr 16, we estimate its total stellar population in reference to the XLF of the Orion Nebula Cluster (ONC) derived from the Chandra Orion Ultradeep Project (Getman et al. 2005). The limiting $\mathrm{X}$-ray luminosity of our sample is $L_{\mathrm{X}} \sim 30.5 \mathrm{ergs} \mathrm{s}^{-1}$. Excluding the high X-ray luminosity tail, i.e., $L_{\mathrm{X}}>31.5 \mathrm{ergs} \mathrm{s}^{-1}$, which includes about $30 \mathrm{X}$-ray sources, the slope of the $\operatorname{Tr} 16$ XLF is consistent with that of the ONC in the X-ray luminosity range of our sample (Fig. 11). This suggests that our sample represents only the brightest $20 \%$ of the X-ray PMS population in the cluster. We hence estimate the total stellar population of $\operatorname{Tr} 16$ to be 1000-1300. Furthermore, the X-ray luminosities are known to be correlated with stellar masses, as found in the ROSAT studies of the Chamaeleon star forming cloud (Feigelson et al. 1993) and also in the Chandra studies of the ONC (Flaccomio et al. 2003; Preibisch et al. 2005). Comparing the XLF of Tr 16 with the ONC XLF versus stellar mass (Figure 5 in Feigelson et al. 2005), we infer that our sample is about $60 \%$ complete for stars with masses greater than $1 M_{\odot}$, and $40 \%$ complete between $0.3-1 M_{\odot}$. Our deep NIR data covering clusters $\operatorname{Tr} 14$, Tr 16 and Cr 232 probe an even lower mass limit of the stellar population. The analysis of the complete NIR results will be presented elsewhere.

\section{SUMMARY}

We detected 454 X-ray sources in the Chandra data of the Carina Nebula observed in September 1999, of which $94 \%$ have counterparts in our NIR observations. A total of 38 known OB stars are found to be X-ray emitters. Their X-ray luminosities range from $\log L_{\mathrm{X}} \sim 30.5-33.4 \mathrm{ergs} \mathrm{s}^{-1}$, with the Wolf-Rayet star HD 93162 being the strongest with $\log L_{\mathrm{X}}=34.12 \mathrm{ergs} \mathrm{s}^{-1}$. HD 93162 also has a very high X-ray to bolometric luminosity, with $L_{\mathrm{X}} / L_{\mathrm{bol}} \sim-5.39$. The only other early-type star with a high $L_{\mathrm{X}} / L_{\mathrm{bol}}$ is the O8.5V type star Tr 16-22. Both HD 93162 and Tr 16-22 have hard X-ray spectra, suggesting the presence of colliding stellar winds. All other $\mathrm{O}$ and early B (up to B3 type) stars follow the canonical relation, $L_{\mathrm{X}} \sim 10^{-6}-10^{-7} L_{\mathrm{bol}}$. Several mid- to late-B type stars have X-ray luminosities comparable to those typical for $\mathrm{T}$ Tauri stars. Hence, it is possible that the $\mathrm{X}$-ray emission from these mid- and late-B stars originates from low-mass companions. We discovered 16 candidate OB stars which escaped detection in previous optical studies because of their large extinction. These candidate OB stars have the same characteristics as known OB stars in terms of X-ray luminosities and NIR fluxes and colors. If most of them turn out to be bona fide OB stars, this $\sim 50 \%$ increase from the number of known OB stars 
This is an unedited preprint of an article accepted for publication in The Astrophysical Journal. The final published article may differ from this preprint. Copyright 2006 by The American Astronomical Society. Please cite as 'ApJ preprint doi:10.1086/'510184".

would add significantly to the stellar energy budget of the region. The NIR colors of the counterparts suggest the existence of a large population of low-mass PMS stars of CTTS type or WTTS type. Some 180 CTTS candidates are identified, whose X-ray luminosities range between $10^{30}$ and $10^{32} \operatorname{ergs~s}^{-1}$, lower than those for OB stars. A compact group of highly reddened sources, clearly detected in the X-rays and NIR, is found to the south-east of $\operatorname{Tr} 16$. The group is spatially close to an $I R A S$ source and coincident with the dust lane where many mid-IR sources have been identified, which is suggestive of ongoing star formation. This star group is "sandwiched" between two peaks of ${ }^{12} \mathrm{CO}(1-0)$ emission. Such star-cloud morphology is also seen on the periphery of the H II complex in Tr 16 . All this suggests a collect-and-collapse process for triggered star formation. A comparison of the X-ray luminosity function of $\operatorname{Tr} 16$ - which is about $60 \%$ complete for stars with masses of 1-3 $M_{\odot}$ and $40 \%$ complete for $0.3-1 M_{\odot}$-with that of typical young star clusters, suggests a total stellar population of $\sim 1000-1300$ in $\operatorname{Tr} 16$.

This publication makes use of the Chandra observations of the Carina Nebula made in September 1999. We made use of the SIMBAD Astronomical Database to search for optical counterparts for the Chandra X-ray sources. We thank Kate Brooks for providing us with the Carina ${ }^{12} \mathrm{CO}(1-0)$ data, which were obtained with the Mopra Antenna, operated by the Australia Telescope National Facility, CSIRO during 1996-1997. We thank the SAAO staff for their maintenance of the IRSF telescope and their kind hospitality. We would like to thank the anonymous referee for useful suggestions and comments. We also greatfully acknowledge the discussions with M. Tsujimoto. KS would like to thank D. K. Ojha for the valuable discussions. KS, WPC and HTL acknowledge the financial support of the grant NSC94-2112-M-008-017 of the National Science Council of Taiwan.

\section{REFERENCES}

Abbott, D. C., Bieging, J. H., \& Churchwell, E. 1984, ApJ, 280, 671

Albacete Colombo, J. F., Méndez, M., \& Morrell, N. I. 2003, MNRAS, 346, 704

Babel, J., \& Montmerle, T. 1997, A\&A, 323, 121

Bessell, M. S. \& Brett, J. M. 1988, PASP, 100, 1134

Berghöfer, T. W., Schmitt, J. H. M. M., Danner, R., \& Cassinelli, J. P. 1997, A\&A, 322, 167

Bohlin, R. C., Savage, B. D., \& Drake, J. F. 1978, ApJ, 224, 132 
This is an unedited preprint of an article accepted for publication in The Astrophysical Journal. The final published article may differ from this preprint. Copyright 2006 by The American Astronomical Society. Please cite as 'ApJ preprint doi:10.1086/'510184".

Brooks, K. J., Whiteoak, J. B., \& Storey, J. W. V. 1998, PASA, 15, 202

Corcoran, M. F., Rawley, G. L., Swank, J. H., \& Petre R. 1995, ApJ, 445, L121

Corcoran, M. F. 2003, Proceeding of IAU Symposium, 212, 130

Chlebowski, T., Seward, F. D., Swank, J., \& Szymkowiak, A. 1984, ApJ, 281, 665

Chlebowski, T., Harnden, F. R., \& Sciortino, S. 1989, ApJ, 341, 427

Cohen, D. H., Cassinelli, J. P. \& Macfarlane, J. J. 1997, ApJ, 487, 867

Crowther, P. A., Smith, L. J., Hillier, D. J., \& Schmutz, W. 1995, A\&A, 293, 427

Deharveng, L., Zavagno, A., \& Caplan, J. 2005, A\&A, 433, 565

de Jong, J. A., Henrichs, H. F., Kaper, L., Nichols, J. S., Bjorkman, K., Bohlender, D. A., Cao, H., Gordon, K., Hill, G., Jiang, Y., Kolka, I., Morrison, N., Neff, J., O’Neal, D., Scheers, B.,\& Telting, J. H. 2001, A\&A, 368, 601

Elmegreen, B. G., \& Lada, C. J. 1977, ApJ, 214, 725

Evans, N. R., Seward, F. D., Krauss, M. I., Isobe, T., Nichols, J., Schlegel, E. M., \& Wolk, S. J. 2003, ApJ, 589, 509

Evans, N. R., Schlegel, E. M., Waldron, W. L., Seward, F. D., Krauss, M. I., Nichols, J., \& Wolk, S. J. 2004, ApJ, 612, 1065

Feigelson, E. D., \& DeCampli, W. M. 1981, ApJ, 243, L89

Feigelson, E. D., Casanova, S., Montmerle, T., \& Guibert, J. 1993, ApJ, 416, 623

Feigelson, E. D., \& Montmerle, T. 1999, ARA\&A, 37, 363

Feigelson, E. D., Broos, P., Gaffney, J. A., Garmire, G., Hillenbrand, L. A., Pravdo, S. H., Townsley, L., \& Tsuboi, Y. 2002, ApJ, 574, 258

Feigelson, E. D., \& Getman, K. V. 2005, in The Initial Mass Function: Fifty Years Later, ed. E. Corbelli et al. (Dordrecht: Kluwer)

Feigelson, E. D., Getman, K., Townsley, L., Garmire, G., Preibisch, T., Grosso, N., Montmerle, T., Muench, A., \& McCaughrean, M. 2005, ApJS, 160, 379

Feinstein, A. 1983, Ap\&SS, 96, 293 
This is an unedited preprint of an article accepted for publication in The Astrophysical Journal. The final published article may differ from this preprint. Copyright 2006 by The American Astronomical Society. Please cite as 'ApJ preprint doi:10.1086/'510184".

Feinstein, A. 1995, RevMexAA, 2, 57

FitzGerald, M. P. 1970, A\&A, 4, 234

FitzGerald, M. P., \& Mehta, S. 1987, MNRAS, 228, 545

Flaccomio, E., Damiani, F., Micela, G., Sciortino, S., Harnden, F. R., Murray, S. S., \& Wolk, S. J. 2003, ApJ, 582, 398

Getman, K. V., Feigelson, E. D., Townsley, L., Bally, J., Lada, C. J., \& Reipurth, B. 2002, ApJ, 575, 354

Getman, K. V., Flaccomio, E., Broos, P. S., Grosso, N., Tsujimoto, M., Townsley, L., Garmire, G. P., Kastner, J., Li, J., Harnden, F. R., Jr., Wolk, S., Murray, S. S., Lada, C. J., Muench, A. A., McCaughrean, M. J., Meeus, G., Damiani, F., Micela, G., Sciortino, S., Bally, J., Hillenbrand, L. A., Herbst, W., Preibisch, T., \& Feigelson, E. D. 2005, ApJS, 160, 319

Harnden, F. R. Jr., Branduardi, G., Gorenstein, P., Grindlay, J., Rosner, R., Topka, K., Elvis, M., Pye, J. P., \& Vaiana, G. S. 1979, ApJ, 234, L51

Ishibashi, K., Corcoran, M. F., Davidson, K., Swank, J. H., Petre, R., Drake, S. A., Daminelli, A., \& White, S. 1999, ApJ, 524, 983

Johnson, H. L. \& Morgan, W. W. 1953, ApJ, 117, 313

Kaper, L., Henrichs, H. F., Nichols, J. S., \& Telting, J. H. 1999, A\&A, 344, 231

Koornneef, J. 1983, A\&A, 128, 84

Kudritzki, R. P., Palsa, R., Feldmeier, A., Puls, J., \& Pauldrach, A. W. A. 1996 in Röntgenstrahlung from the Universe, ed. H. U. Zimmermann, J. Trümper, \& H. Yorke (Garching:MPE), 9

Levato, H. \& Malaroda, S. 1981, PASP, 93, 714

Lucy, L. B., \& White, R. L. 1980, ApJ, 241, 300

Lucy, L. B. 1982, ApJ, 255, 286

Massey, P., \& Johnson, J. 1993, AJ, 105, 980

Massey, P., DeGioia-Eastwood, K. \& Waterhouse, E. 2001, AJ, 121, 1050 
This is an unedited preprint of an article accepted for publication in The Astrophysical Journal. The final published article may differ from this preprint. Copyright 2006 by The American Astronomical Society. Please cite as 'ApJ preprint doi:10.1086/'510184".

Maggio, A., Sciortino, S., Vaiana, G. S., Majer, P., Bookbinder, J., Golub, L., Harnden, F. R. Jr., \& Rosner, R. 1987, ApJ, 315, 687

Megeath, S. T., Cox, P., Bronfman, L., \& Roelfsema, P. R. 1996, A\&A, 305, 296

Meyer, M., Calvet, N., \& Hillenbrand, L. A. 1997, AJ, 114, 288

Morrell, N., Garcia, B., \& Levato, H. 1988, PASP, 100, 1431

Nagayama, T., Nagashima, C., Nakajima, Y., Nagata, T., Sato, S., Nakaya, H., Yamamuro, T., Sugitani, K., \& Tamura, M. 2003, Proc. SPIE, 4841, 459

Nakajima, Y., Kato, D., Nagata, T., Tamura, M., Sato, S., Sugitani, K., Nagashima, C., Nagayama, T., Iwata, I., Ita, Y., Tanabe, T., Kurita, M., Nakaya, H., \& Baba, D. 2005, AJ, 129, 776

Owocki, S. P., \& Cohen, D. H. 1999, ApJ, 520, 833

Pallavicini, R., Golub, L., Rosner, R., Vaiana, G. S., Ayres, T. \& Linsky, J. L. 1981, ApJ, 248,279

Persson, S. E., Murphy, D. C., Krzeminski, W., Roth, M., \& Rieke, M. J. 1998, AJ, 116, 2475

Preibisch, T., \& Zinnecker, H. 2002, AJ, 123, 1613

Preibisch, T., Kim, Y.C., Favata, F., Feigelson, E. D., Flaccomio, E., Getman, K., Micela, G., Sciortino, S., Stassun, K., Stelzer, B., \& Zinnecker, H. 2005, ApJS, 160, 401

Rieke, G. H., \& Lebofsky, M. J., 1985, ApJ, 288, 618

Schulz, N. S., Canizares, C., Huenemoerder, D., \& Tibbets, K. 2003, ApJ, 595, 365

Seward, F. D., Butt, Y. M., Karovska, M., Prestwich, A., Schlegel, E. M., \& Corcoran, M. 2001, ApJ, 553, 832

Smith, N., Egan, M. P., Carey, S., Price, S. D., Morse, J. A., Price, P. A. 2000, ApJ, 532, L145

Smith, R. G. 1987, MNRAS, 227, 943

Stetson, P. B. 1987, PASP, 99, 191

Stelzer, B., Flaccomio, E., Montmerle, T., Micela, G., Sciortino, S., Favata, F., Preibisch, T., \& Feigelson, E. D. 2005, ApJS, 160, 557 
This is an unedited preprint of an article accepted for publication in The Astrophysical Journal. The final published article may differ from this preprint. Copyright 2006 by The American Astronomical Society. Please cite as 'ApJ preprint doi:10.1086/'510184".

Tapia, M., Roth, M., Marraco, H., \& Ruiz, M. T. 1988, MNRAS, 232, 661

Tapia, M., Roth, M., Vázquez, R. A., \& Feinstein, A. 2003, MNRAS, 339, 44

Townsley, L. K., Broos, P. S., Feigelson, E. D., Garmire, G. P., \& Getman, K. V. 2006, AJ, 131,2164

Turner, D. G., \& Moffat, A. F. J. 1980, MNRAS, 192, 283

ud-Doula, A., \& Owocki, S. P. 2002, ApJ, 576, 413

van der Hucht, K. A. 2001, NewARev., 45, 135

van Loo, S., Runacres, M. C., \& Blomme, R. 2004, A\&A, 418, 717

Walborn, N. R. 1973, AJ, 78, 1067

Walborn, N. R. 1982, AJ, 87, 1300

Walborn, N. R. 1995, RevMexAA 2, 51

Walter, F. M., \& Kuhi, L. V. 1984, ApJ, 284, 194

Walter, F. M., \& Barry, D. C. 1991, in The Sun in Time, eds. C. P. Sonnett, M. S. Giampa \& M. S. Matthews (Tucson: Univ. Arizona Press), 633

Whitworth, A. P., Bhattal, A. S., Chapman, S. J., Disney, M. J., \& Turner, J. A. 1994, MNRAS, 268, 291

Zavagno, A., Deharveng, L., Comerón, F., Brand, J., Massi, F., Caplan, J., \& Russeil, D. 2006, A\&A, 446, 171

This preprint was prepared with the AAS LATEX macros v5.2. 
This is an unedited preprint of an article accepted for publication in The Astrophysical Journal. The final published article may differ from this preprint. Copyright 2006 by The American Astronomical Society. Please cite as 'ApJ preprint doi:10.1086/'510184".

Table 1. CHANDRA OBSERVATIONS

\begin{tabular}{ccc}
\hline \hline Observation ID & 50 & 1249 \\
\hline Pointing & $10^{h} 45^{m} 03^{s} .60-59^{\circ} 41^{\prime} 03^{\prime \prime}$ & $10^{h} 45^{m} 03^{s} .60-59^{\circ} 41^{\prime} 03^{\prime \prime}$ \\
Roll Observed & $176^{\circ}$ & $176^{\circ}$ \\
Start Date & $1999-09-0619: 48: 12$ & $1999-09-0623: 45: 34$ \\
Exposure Time & $12.04 \mathrm{ks}$ & $9.75 \mathrm{ks}$ \\
Reduced Exposure Time & $8545.99 \mathrm{~s}$ & $9574.01 \mathrm{~s}$ \\
\hline
\end{tabular}

Note. - see text for the IRSF observation log. 
This is an unedited preprint of an article accepted for publication in The Astrophysical Journal. The final published article may differ from this preprint. Copyright 2006 by The American Astronomical Society. Please cite as 'ApJ preprint doi:10.1086/'510184"'.

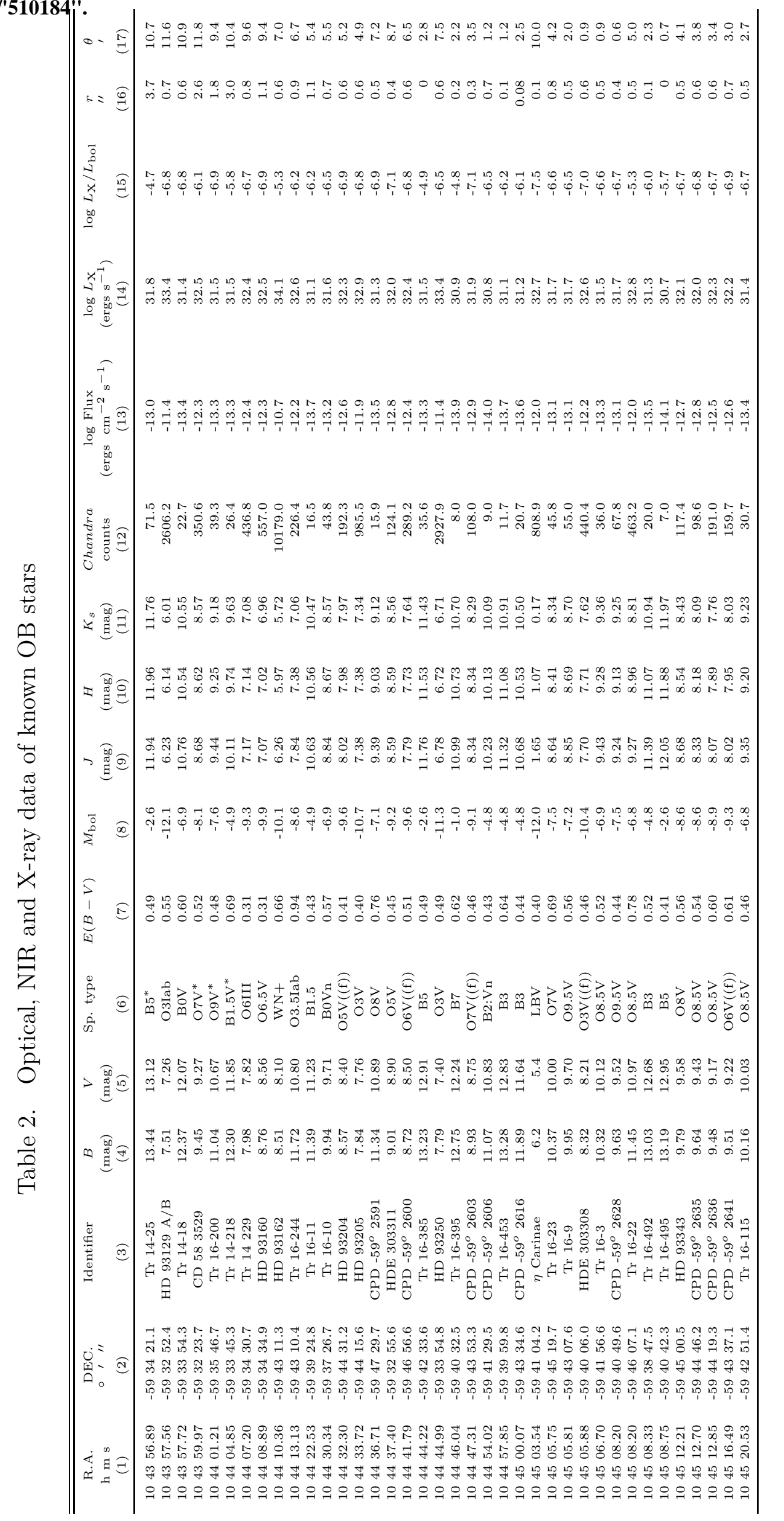


This is an unedited preprint of an article accepted for publication in The Astrophysical Journal. The final published article may differ from this preprint. Copyright 2006 by The American Astronomical Society. Please cite as 'ApJ preprint doi:10.1086/'510184".

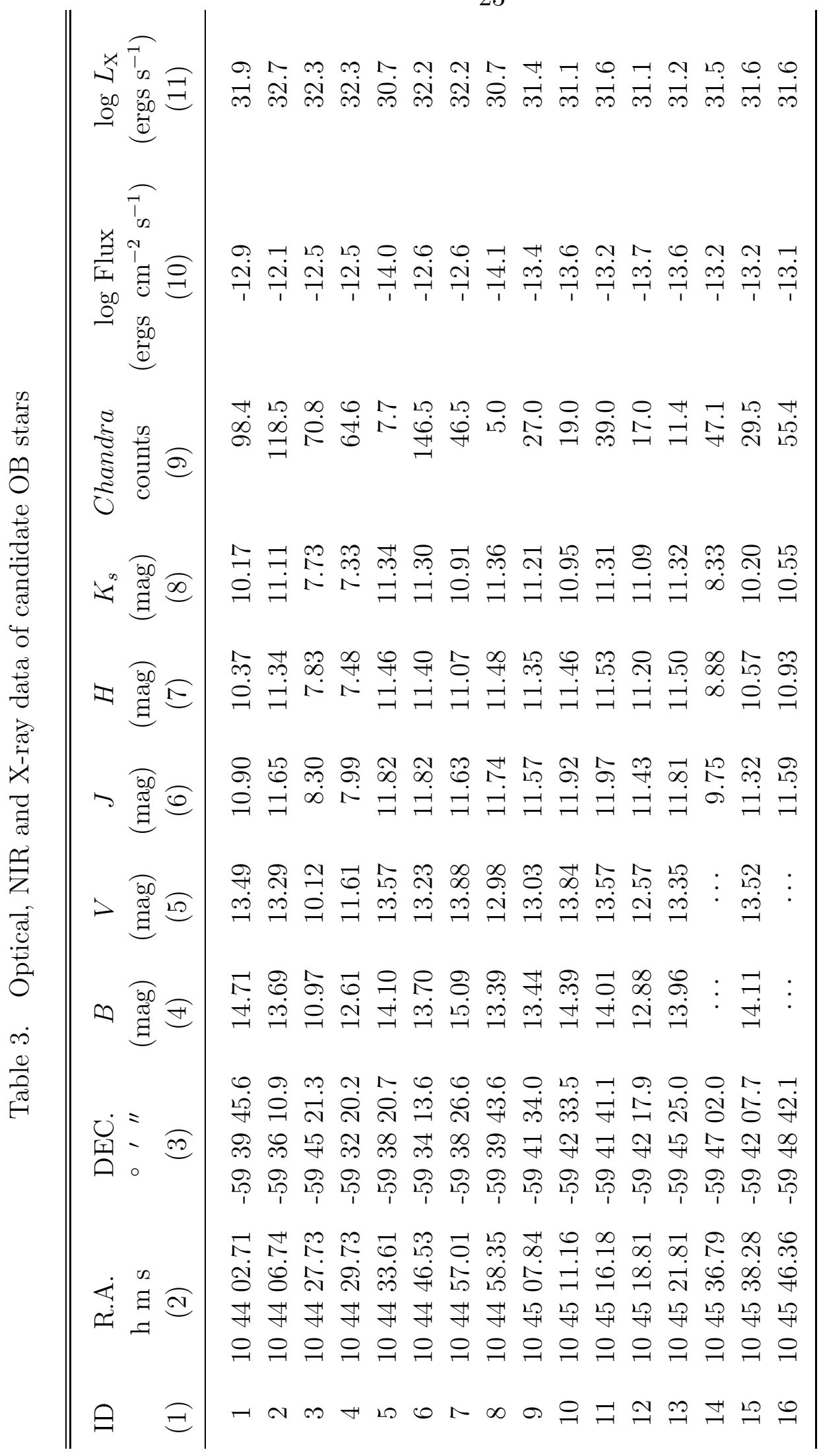


This is an unedited preprint of an article accepted for publication in The Astrophysical Journal. The final published article may differ from this preprint. Copyright 2006 by The American Astronomical Society. Please cite as 'ApJ preprint doi:10.1086/'510184". 
This is an unedited preprint of an article accepted for publication in The Astrophysical Journal. The final published article may differ from this preprint. Copyright 2006 by The American Astronomical Society. Please cite as 'ApJ preprint doi:10.1086/'510184".

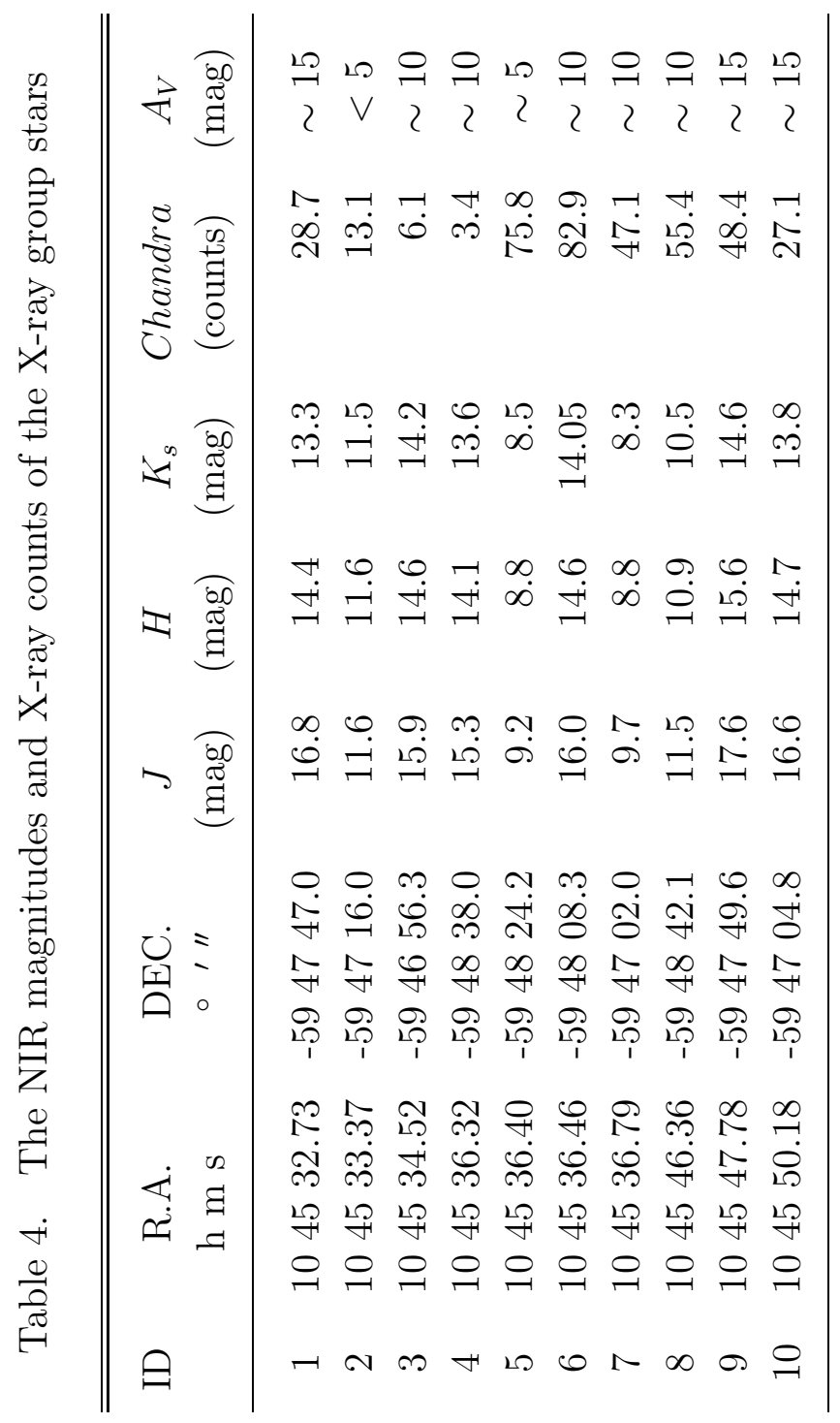


This is an unedited preprint of an article accepted for publication in The Astrophysical Journal. The final published article may differ from this preprint. Copyright 2006 by The American Astronomical Society. Please cite as 'ApJ preprint doi:10.1086/'510184".

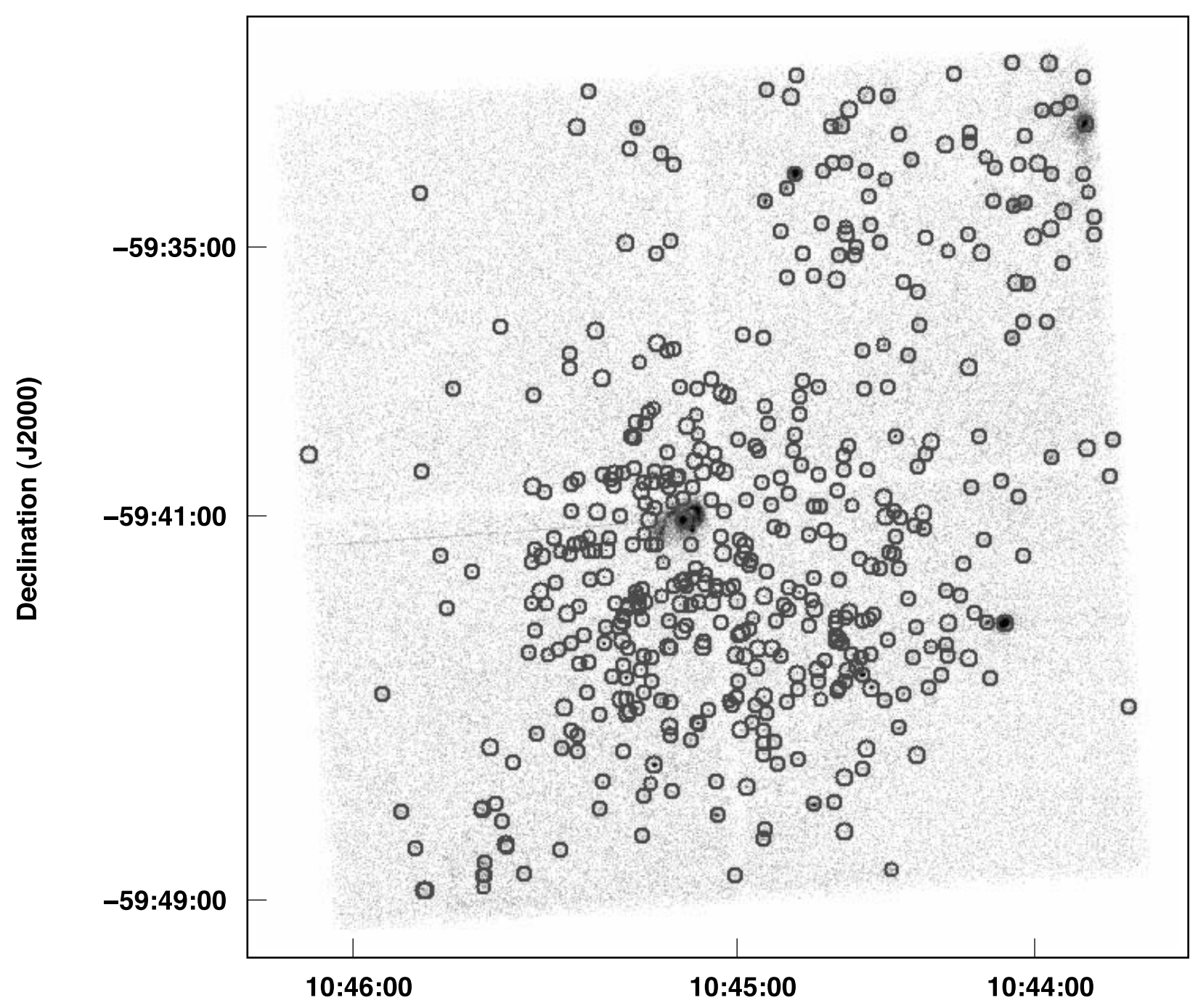

Right Ascention (J2000)

Fig. 1.- The Chandra image of the Carina Nebula. It is merged from two observations (obsIDs 1249 and 50), taken by the Chandra ACIS-I, centered on $\eta$ Carinae, with a field of view of $17^{\prime} \times 17^{\prime}$. The point sources are marked as circles with equal size radius, though the off-axis sources have larger positional uncertainties. 
This is an unedited preprint of an article accepted for publication in The Astrophysical Journal. The final published article may differ from this preprint. Copyright 2006 by The American Astronomical Society. Please cite as 'ApJ preprint doi:10.1086/'510184"'.

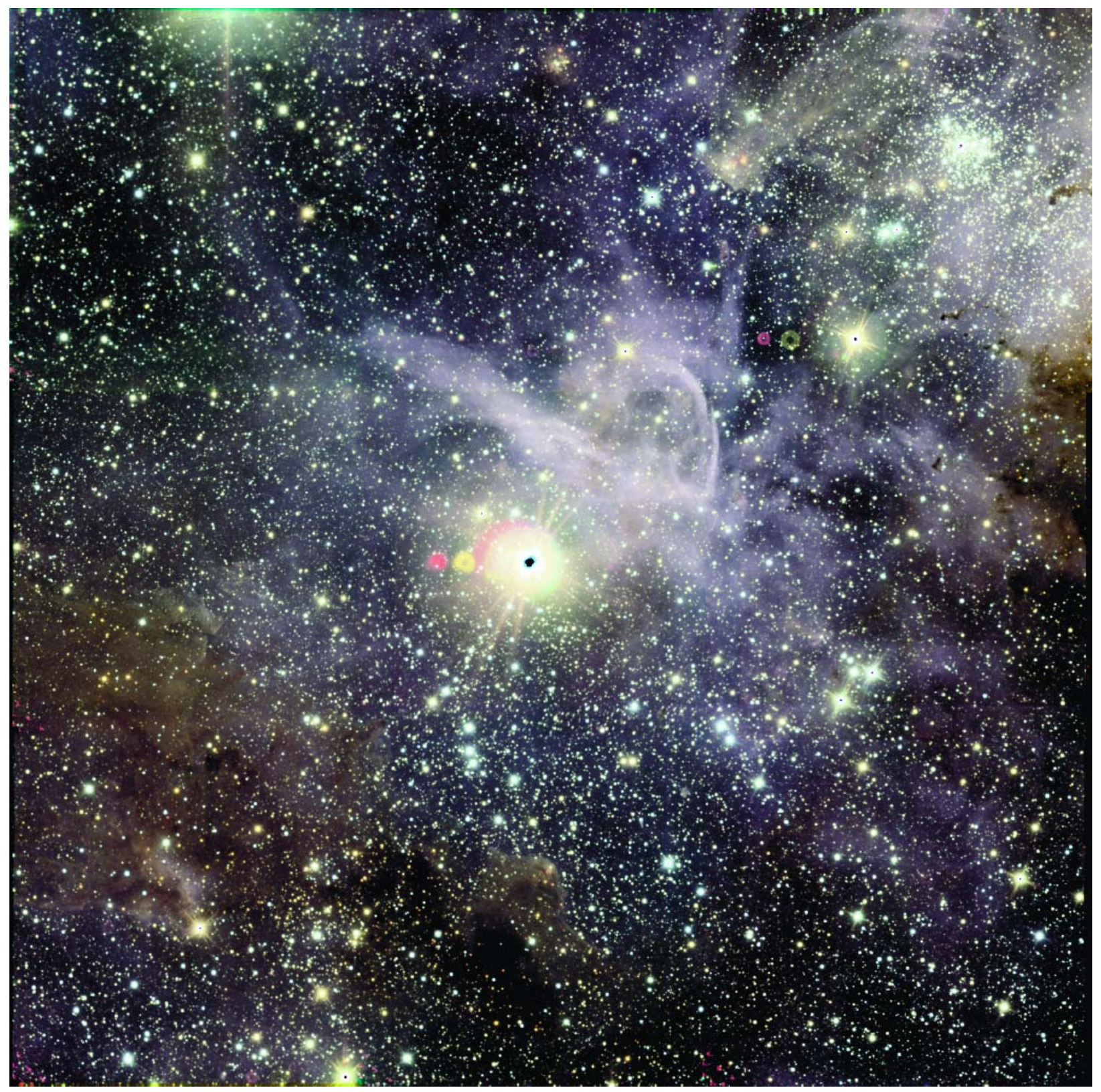

Fig. 2.- The IRSF CMYK (cyan, magenta, yellow and black) color-composite image of the Carina Nebula. The image is centered on $\eta$ Carinae and has a field of view of $\sim 22^{\prime} \times 22^{\prime}$, with north to the top and east to the left. Tr 16 can be seen in the center, whereas $\operatorname{Tr} 14$ is located to the north-west. 
This is an unedited preprint of an article accepted for publication in The Astrophysical Journal. The final published article may differ from this preprint. Copyright 2006 by The American Astronomical Society. Please cite as 'ApJ preprint doi:10.1086/'510184".

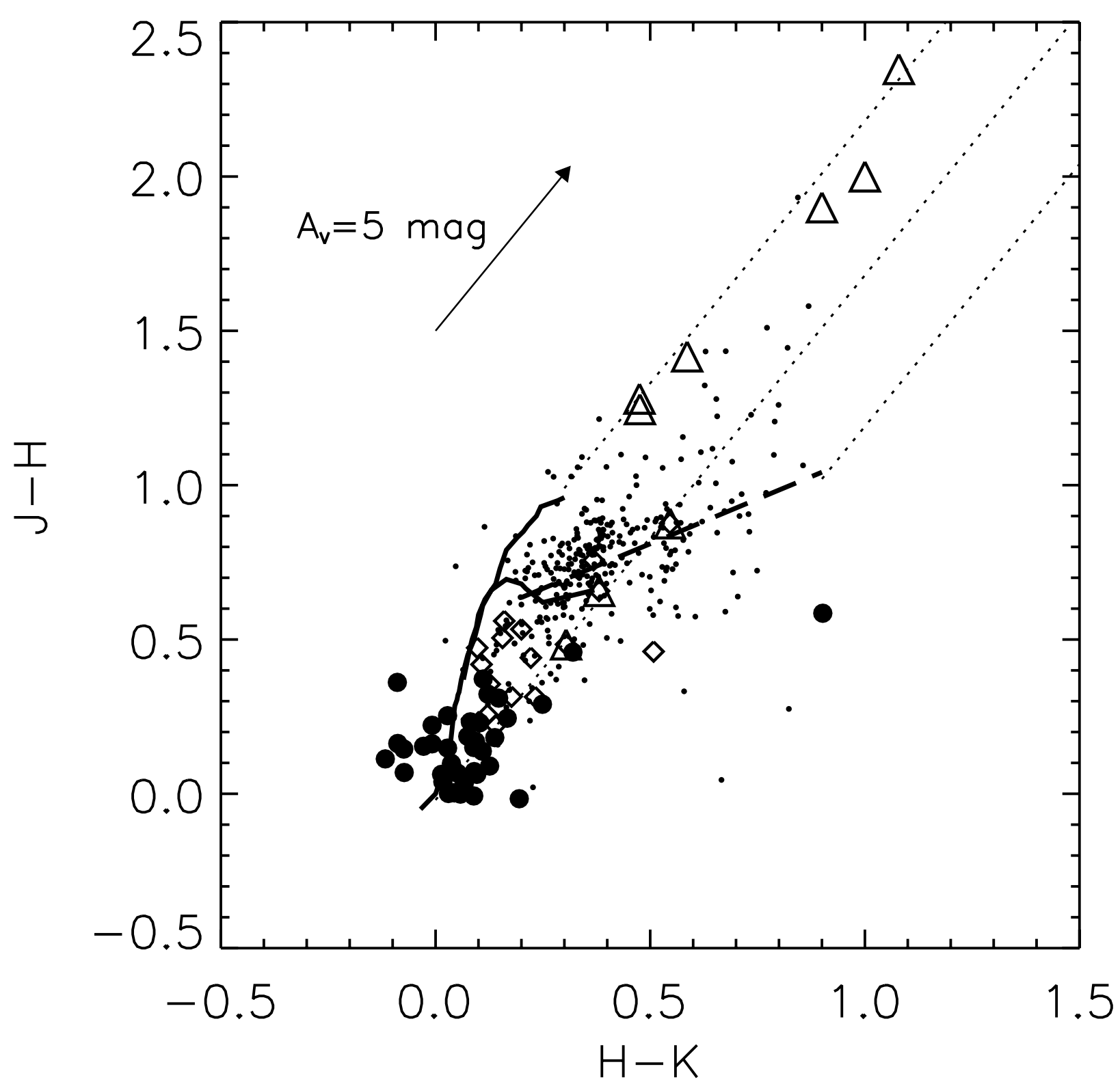

Fig. 3.- The NIR color-color diagram of all X-ray sources. The known OB stars are shown as filled circles, the candidate OB stars are shown as diamonds and the members of an embedded X-ray group (see text) are shown as triangles. All other X-ray sources are shown as dots. The dwarf and giant loci are shown as the solid curves (Bessell \& Brett 1988) and the classical T Tauri locus is shown as the dashed line (Meyer et al. 1997). The dotted lines represent the reddening band. The slope of the reddening vector is 1.7. The known OB star with very red color, $H-K \sim 0.9$, is $\eta$ Carinae. 
This is an unedited preprint of an article accepted for publication in The Astrophysical Journal. The final published article may differ from this preprint. Copyright 2006 by The American Astronomical Society. Please cite as 'ApJ preprint doi:10.1086/'510184".

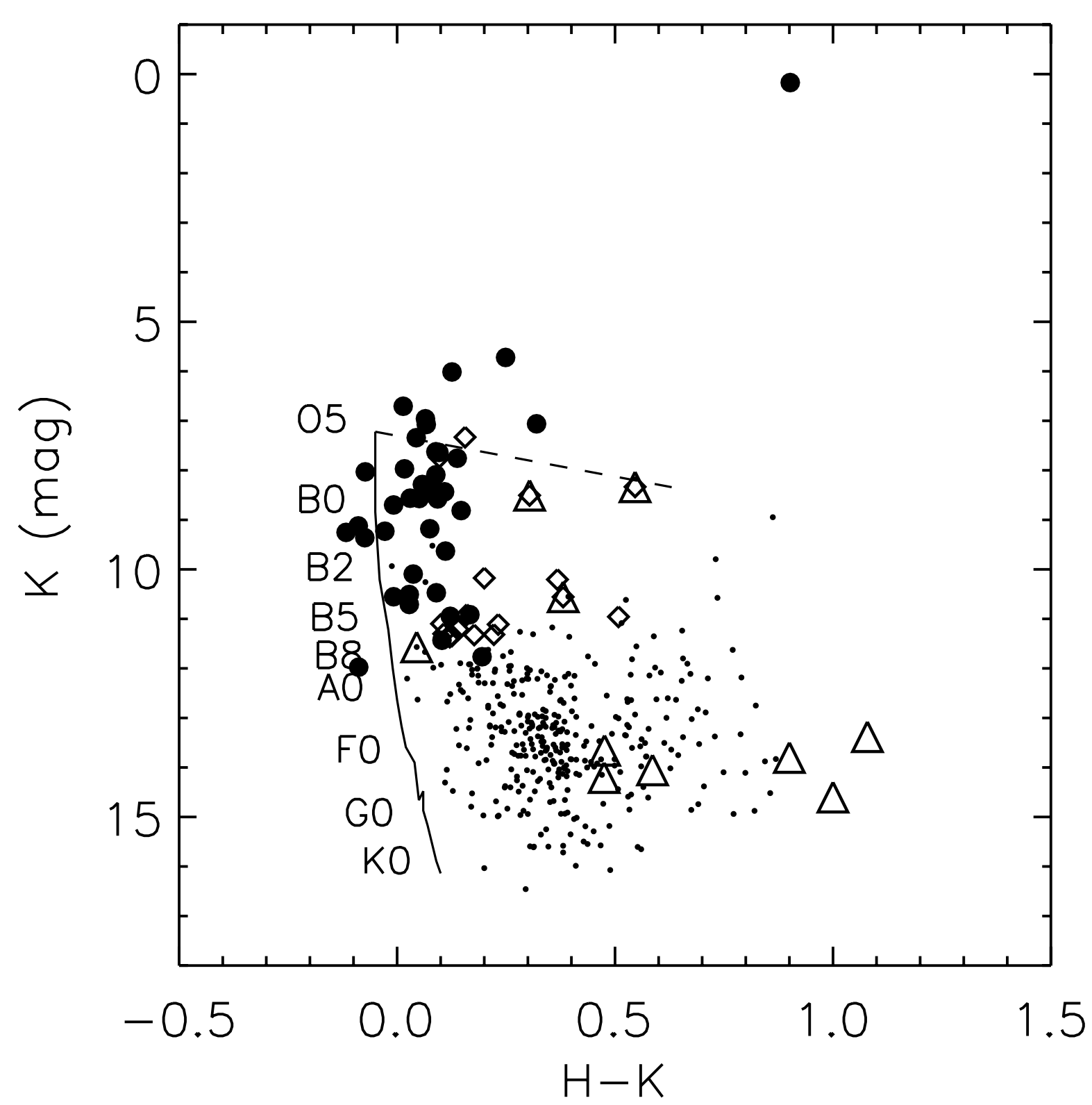

Fig. 4.- The NIR color-magnitude diagram of all X-ray sources. The known OB stars are shown as filled circles, the candidate OB stars are shown as diamonds and the members of an embedded X-ray group are shown as triangles. All other sources are shown as dots. The vertical line represents the unreddened main sequence (Koornneef 1983) at $2.5 \mathrm{kpc}$ and the dashed line represents the reddening vector for $A_{V}=10$ mag. 
This is an unedited preprint of an article accepted for publication in The Astrophysical Journal. The final published article may differ from this preprint. Copyright 2006 by The American Astronomical Society. Please cite as 'ApJ preprint doi:10.1086/'510184".

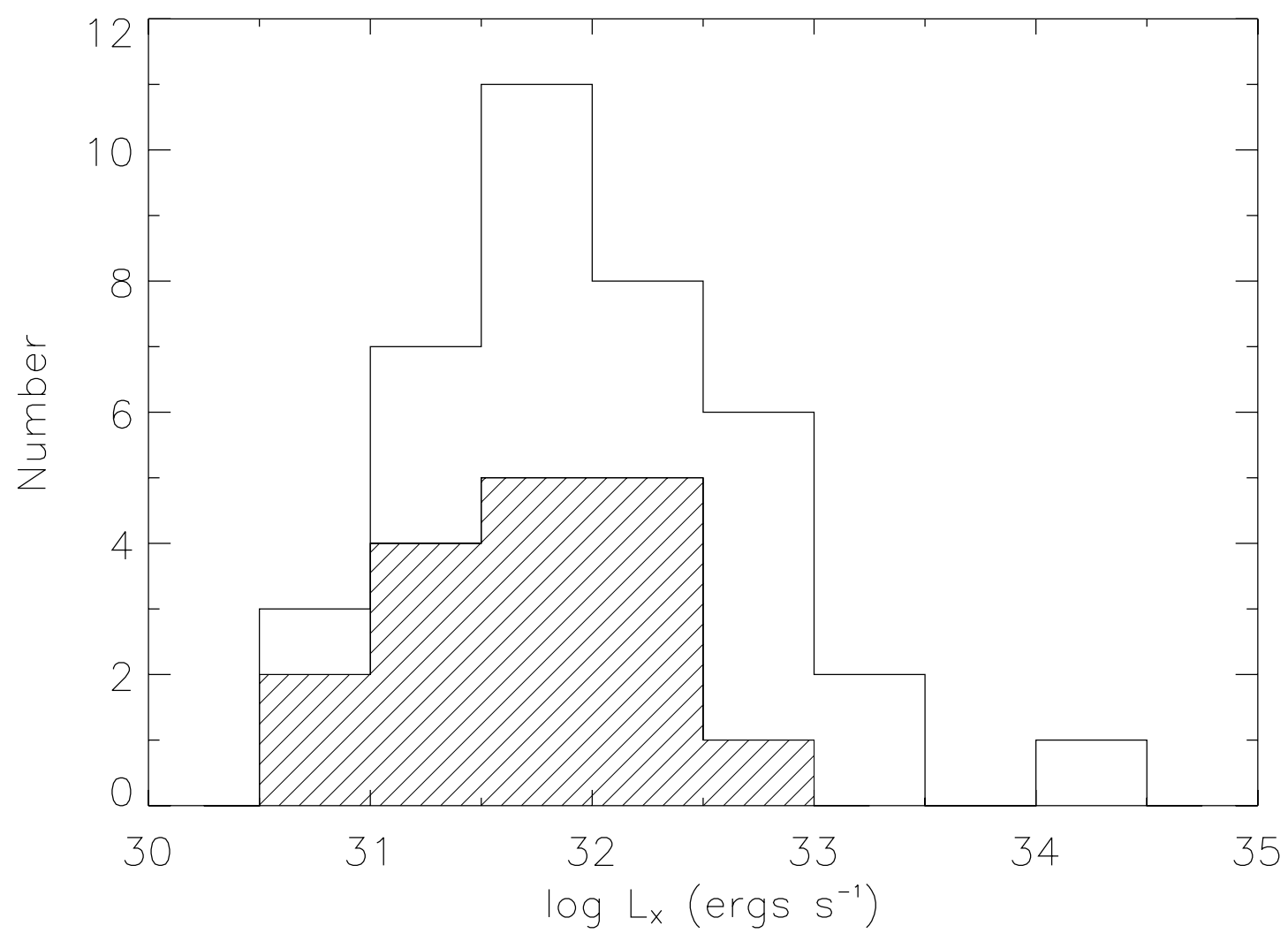

Fig. 5.- The distribution of X-ray luminosities of the known OB stars (unshaded) and the candidate OB stars (shaded). 
This is an unedited preprint of an article accepted for publication in The Astrophysical Journal. The final published article may differ from this preprint. Copyright 2006 by The American Astronomical Society. Please cite as 'ApJ preprint doi:10.1086/'510184'".

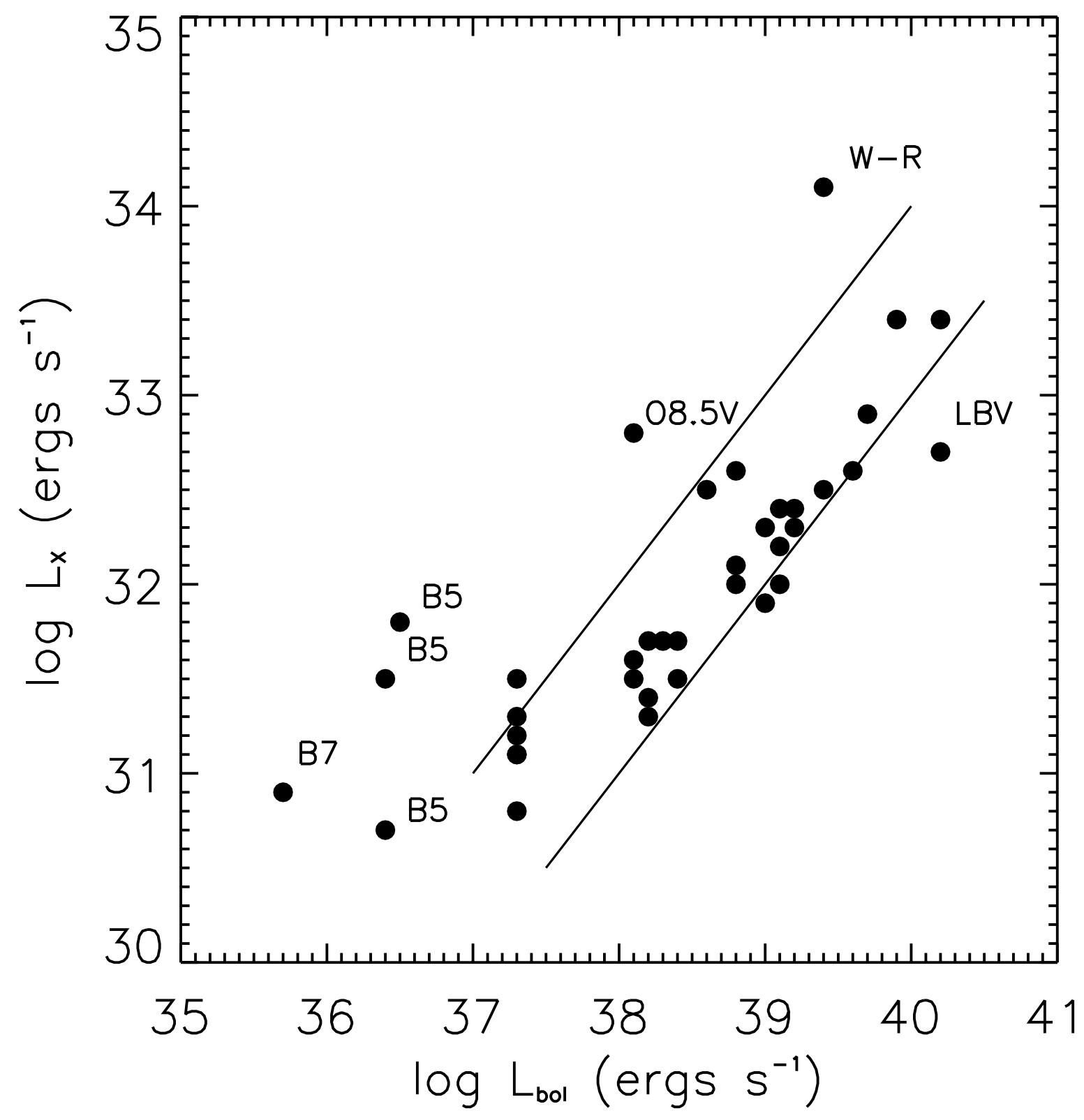

Fig. 6.- The X-ray luminosity versus stellar bolometric luminosity. Most OB stars fall between the two lines, corresponding to $\log \left(L_{\mathrm{X}} / L_{\mathrm{bol}}\right)$ equal to -6 and -7 . The outliers are labeled with their spectral types. 
This is an unedited preprint of an article accepted for publication in The Astrophysical Journal. The final published article may differ from this preprint. Copyright 2006 by The American Astronomical Society. Please cite as 'ApJ preprint doi:10.1086/'510184".

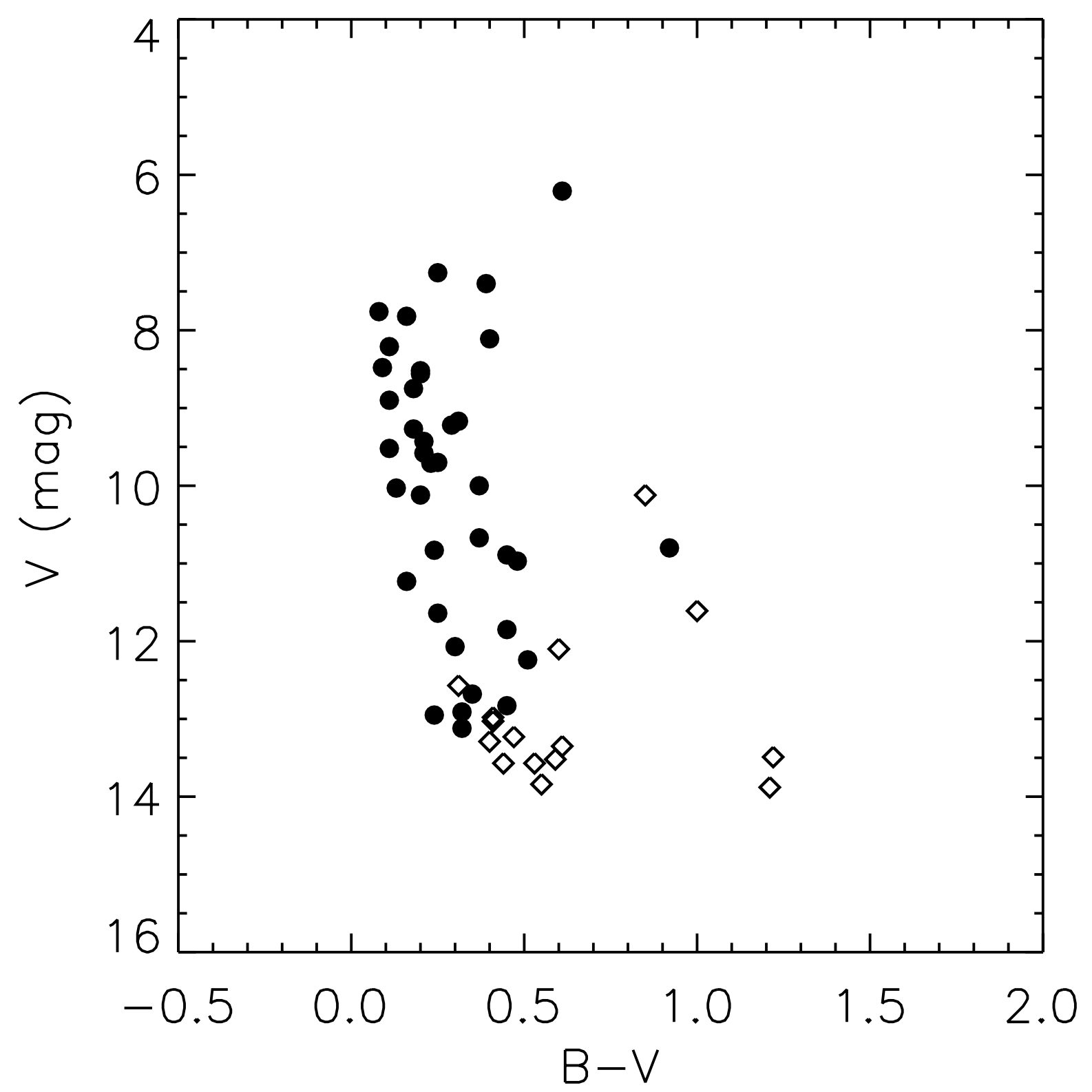

Fig. 7.- The optical color-magnitude diagram of the known OB stars (filled circles) and candidate OB stars (diamonds). 
This is an unedited preprint of an article accepted for publication in The Astrophysical Journal. The final published article may differ from this preprint. Copyright 2006 by The American Astronomical Society. Please cite as 'ApJ preprint doi:10.1086/'510184".

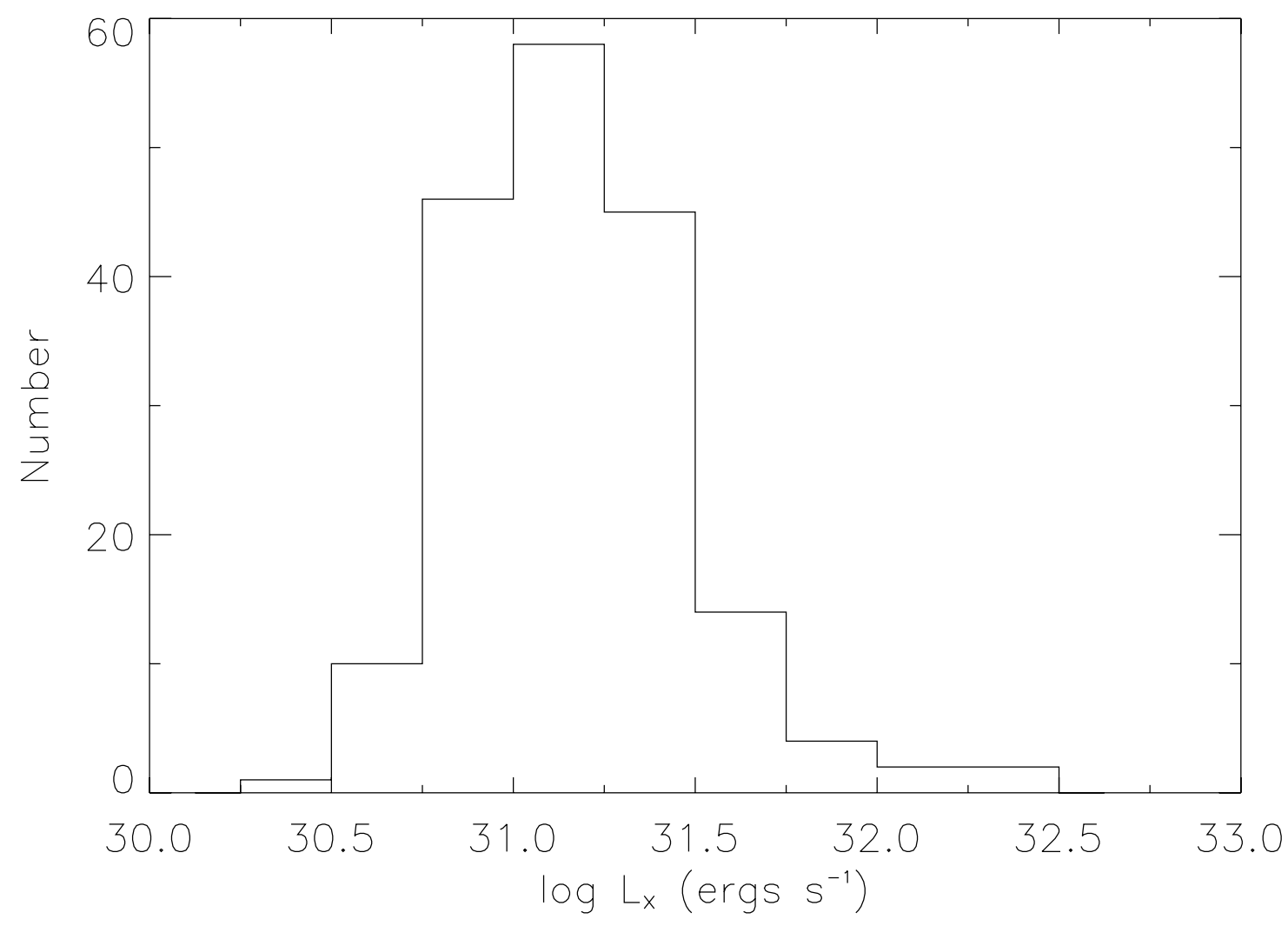

Fig. 8. - The distribution of X-ray luminosities of the T Tauri candidates selected by the criteria given in Meyer et al. (1997). 
This is an unedited preprint of an article accepted for publication in The Astrophysical Journal. The final published article may differ from this preprint. Copyright 2006 by The American Astronomical Society. Please cite as 'ApJ preprint doi:10.1086/'510184".

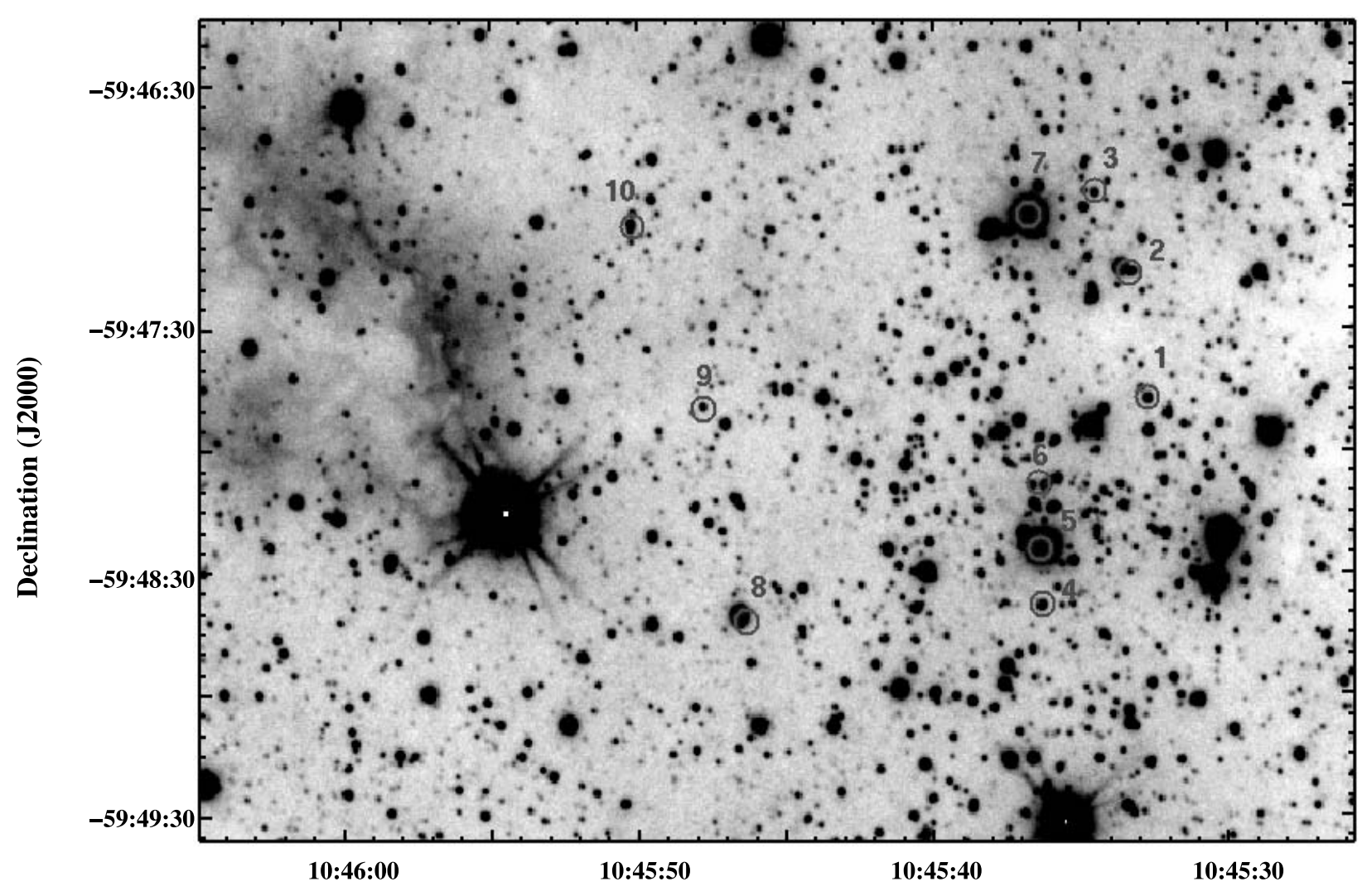

Right Ascention (J2000)

Fig. 9.- The IRSF $K_{s}$ image of the compact X-ray group with north to the top and east to the left. The field of view is $\sim 3^{\prime} \times 5^{\prime}$. The X-ray sources are marked and labeled by their identification number in Table 4. 
This is an unedited preprint of an article accepted for publication in The Astrophysical Journal. The final published article may differ from this preprint. Copyright 2006 by The American Astronomical Society. Please cite as 'ApJ preprint doi:10.1086/'510184".

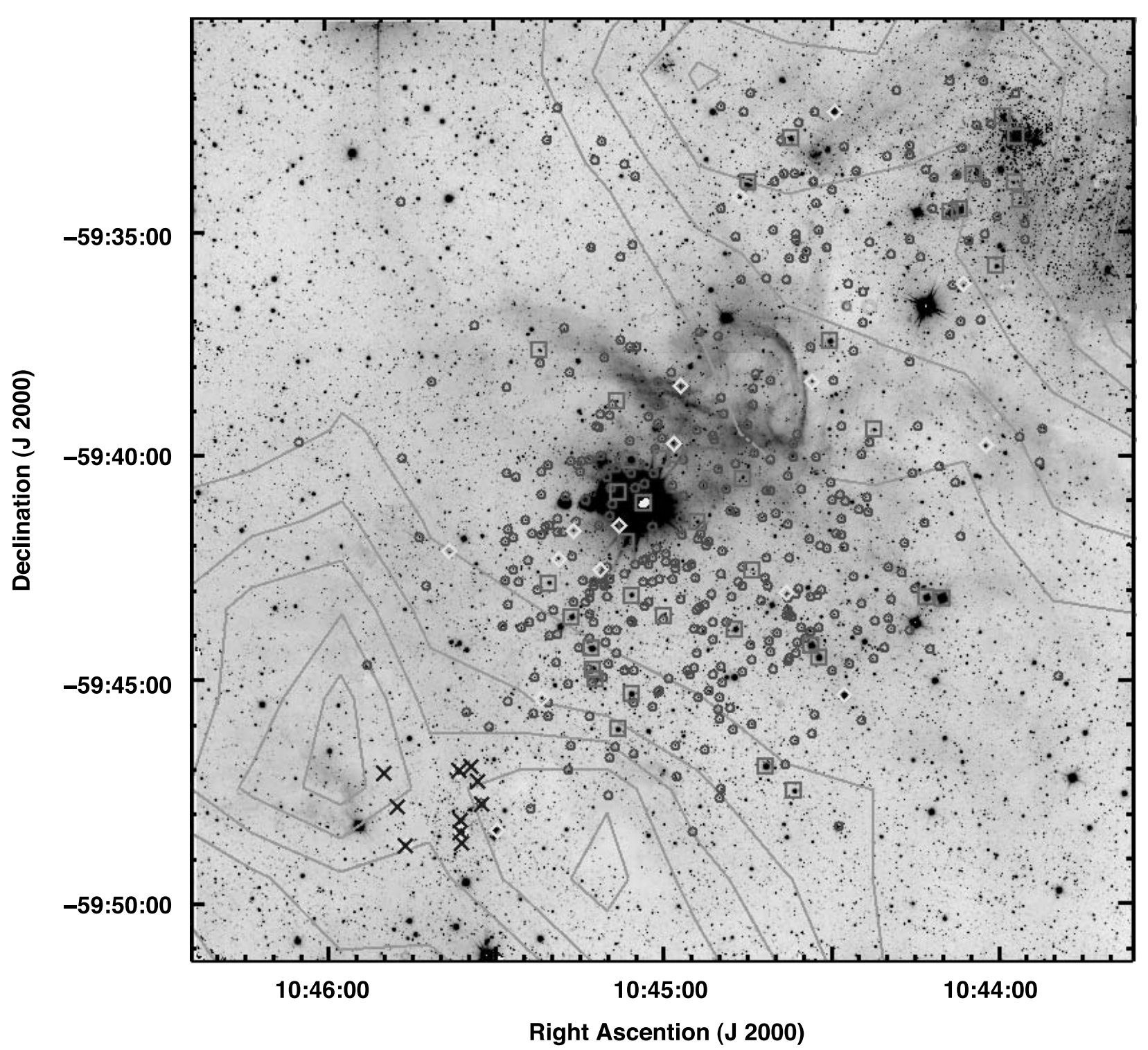

Fig. 10. - The Chandra sources overlaid on the mosaic $K_{s}$ band image, centered on $\eta$ Carinae. Also shown, as contours, are the ${ }^{12} C O(1-0)$ emission (Brooks et al. 1998). The known OB stars are marked as boxes and the candidate OB stars are marked as diamonds. The stars of the embedded X-ray group to the south-east of $\operatorname{Tr} 16$ are marked as crosses, whereas all other Chandra sources that do not fall in any of the above categories are marked as circles. 
This is an unedited preprint of an article accepted for publication in The Astrophysical Journal. The final published article may differ from this preprint. Copyright 2006 by The American Astronomical Society. Please cite as 'ApJ preprint doi:10.1086/'510184".

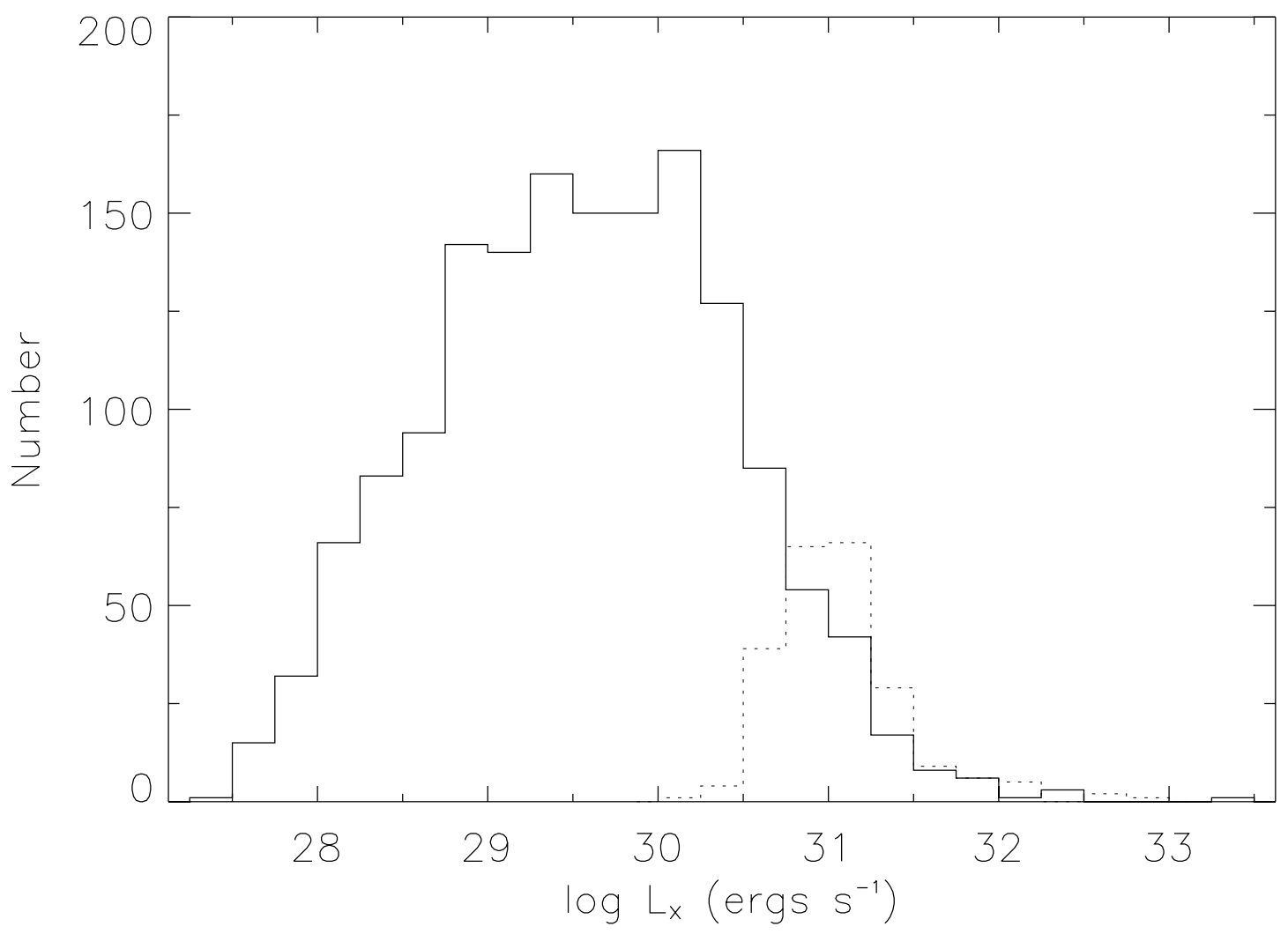

Fig. 11. - Comparison of the Tr 16 XLF (in dashed lines, this work) with the ONC XLF (Getman et al. 2005). 